Miniaturization Technologies

November 1991

OTA-TCT-514

NTIS order \#PB92-150325

\section{MINIATURIZATION TECHNOLOGIES}

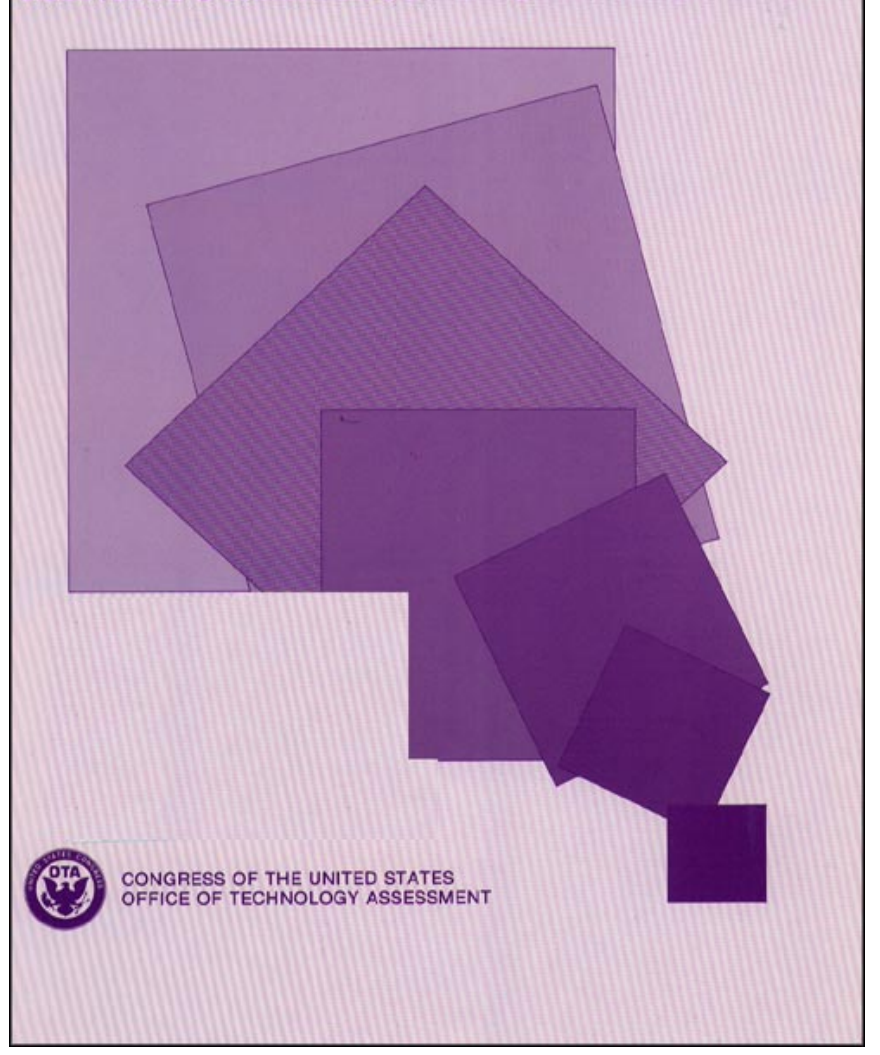




\section{Recommended Citation:}

U.S. Congress, Office of Technology Assessment, Miniaturization Technologies, OTA-TCT514 (Washington, DC: U.S. Government Printing Office, November 1991).

For sale by the U.S. Government Printing Office

Superintendent of Documents, Mail Stop: SSOP, Washington, DC 20402-9328

ISBN 0-16-035983-X 


\section{Foreword}

Advances in miniaturization technologies have had dramatic impacts on our lives. Radios, computers, and telephones that once occupied large volumes now fit in the palm of a hand. Dozens of sensors are sent on spacecraft to the planets and on instruments into the human body. Electronic brains are in everything from bombs to washing machines.

This report analyzes various technologies that may be important for future advances in miniaturization. Current research in the United States and other nations is pushing the limits of miniaturization to the point that structures only hundreds of atoms thick will be commonly manufactured. Researchers studying atomic and molecular interactions are continuing to push the frontiers, creating knowledge needed to continue progress in miniaturization. Scientists and engineers are creating microscopic mechanical structures and biological sensors that will have novel and diverse applications.

OTA characterizes U.S. research and development in miniaturization technologies as the best in the world. Despite the growing prowess of foreign research, American researchers continue to innovate and push the frontiers of miniaturization. The more elusive challenge is to translate success in the laboratory to success in the global marketplace.

OTA gratefully acknowledges the contributions of the workshop participants, contractors, reviewers, and contributors who provided information, advice, and assistance. OTA, of course, bears sole responsibility for the contents of this report.

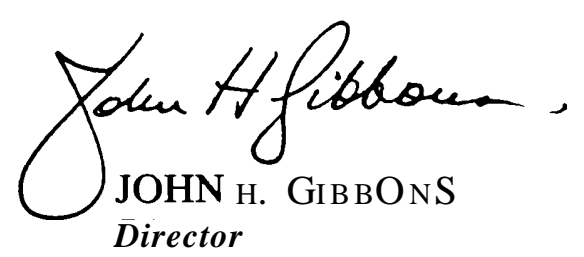




\author{
Karl Hess, Chairman \\ Professor of Electrical Engineering \\ Beckman Institute \\ University of Illinois
}

Fernand Bedard

Research Physicist

National Security Agency

T.H. Phillip Chang

IBM T.J. Watson Research Center

Harold Craighead

Director

National Nanofabrication Facility

Cornell University

Eric Drexler

Visiting Scholar

Stanford Computer Science Department

Stanford University

David K. Ferry

Regents Professor

Department of Electrical Engineering

Arizona State University

Kaigham Gabriel

AT\&T Bell Labs

Stephen Jacobsen

Director

Center of Engineering Design

University of Utah
Wen Hsiung Ko

Professor of Electrical Engineering

Professor of Biomedical Engineering

Member of the Electronics Design Center

Case Western Reserve University

Carl Kukkonen

Director

Center for Space Microelectronics Technology

Jet Propulsion Laboratory

Richard S. Muller

Department of Electrical Engineering/ Computer Science

University of California at Berkeley

James S. Murday

Superintendent of Chemistry

Naval Research Laboratory

Richard Potember

Johns Hopkins Applied Physics Lab

Arati Prabhakar

Director

Microelectronics Technology Office

DARPA

George Sai-Halasz

Research Staff Member

IBM T.J. Watson Research Center

NOTE: OTA appreciates and is grateful for the valuable assistance and thoughtful critiques provided by the workshop participants. The workshop participants do not, however, necessarily approve, disapprove, or endorse this report. OTA assumes full responsibility for the report and the accuracy of its contents. 


\section{Miniaturization Technologies Project Staff}

John Andelin, Assistant Director, OTA

Science, Information, and Natural Resources Division

James W. Curlin, Program Manager

Telecommunication and Computing Technologies Program

Project Staff

Sunil Paul, Project Director

\section{Contractor Staff}

Harold Craighead, Cornell University

Michael Skvarla, Cornell University

Clayton Teague, National Institute of Standards and Technology

Administrative Staff

Liz Emanuel, Office Administrator

Jo Anne Young, Secretary

Karolyn St. Clair, PC Specialist 


\section{Miniaturization Technologies}

\section{Reviewers}

John Alic

Industry, Technology and

Employment Program

Office of Technology Assessment

Samuel Baldwin

Energy and Materials Program

Office of Technology Assessment

Robert Bate

Texas Instruments

Fernand Bedard

National Security Agency

Robert Birge

Center for Molecular Electronics

Syracuse University

Frederico Capasso

AT\&T Bell Labs

Kenneth Davis

Electronics Division

Office of Naval Research

Bob Dennard

IBM

Eric Drexler

Stanford Computer Science

Department

Stanford University

Larry Dworsky

Motorola, Inc.

David K. Ferry

Arizona State University

Kaigham Gabriel

AT\&T Bell Labs

Joseph Giachino

Ford Motor Co.

L Val Giddings

U.S. Department of Agriculture
Lance Glasser

DARPA

Henry Guckel

University of WisconsinMadison

George Hazelrigg

Electrical and Communications Systems Division

National Science Foundation

Karl Hess

University of Illinois

Stephen Jacobsen

Center of Engineering Design

University of Utah

Wen Hsiung Ko

Electrical Engineering and Applied Physics

Case Western Reserve University

Carl Kukkonen

Center for Space Microelectronics Technology

Jet Propulsion Laboratory

Rolf Landauer

IBM

Ralph Merkle

Xerox Palo Alto Research Center

Lester W. Milbrath

State University of New York at Buffalo

Richard S. Muller

Department of Electrical Engineering/Computer Science

University of California at Berkeley

James S. Murday

Naval Research Laboratory
Robyn Nishimi

Biological Applications Program

Office of Technology Assessment

Wally Parce

Molecular Devices

Carl Pilcher

Office of Space Science and Applications

National Aeronautics and Space Administration

Richard Potember

Johns Hopkins Applied Physics Lab

Arati Prabhakar

DARPA

George Sai-Halasz

IBM T.J. Watson Research Center

Charles Shanley

Motorola, Inc.

Clayton Teague

NIST

William Trimmer

Princeton University

William Troutman

AT\&T

Matthew Weinberg

Energy and Materials Program

Office of Technology Assessment

Sheryl Winston

Biological Applications Program

Office of Technology Assessment

Ken D. Wise

The University of Michigan

NOTE: OTA appreciates and is grateful for the valuable assistance and thoughtful critiques provided by the reviewers. The reviewers do not, however, necessarily approve, disapprove, or endorse this report. OTA assumes full responsibility for the report and the accuracy of its contents. 


\section{Contents}

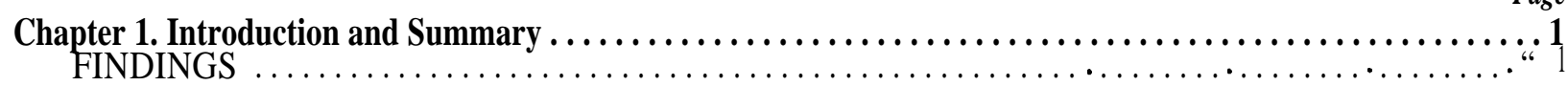

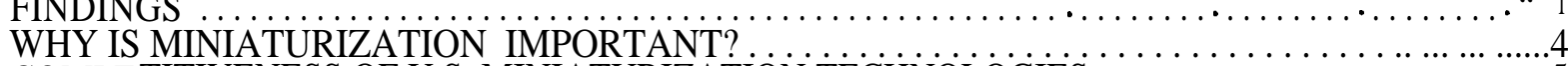

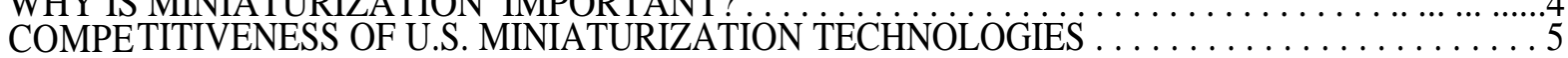

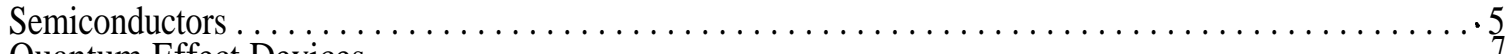

Quantum Effect Devices ..........................................

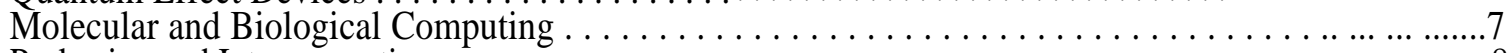

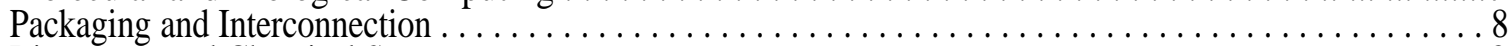

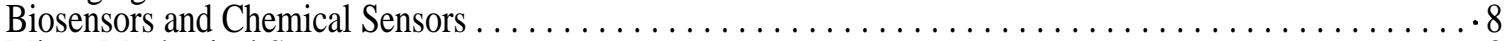

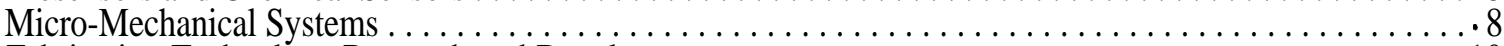

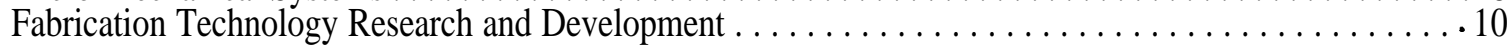

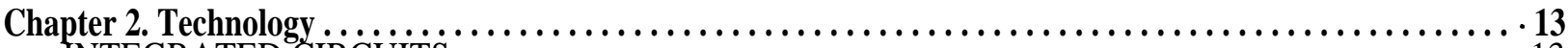

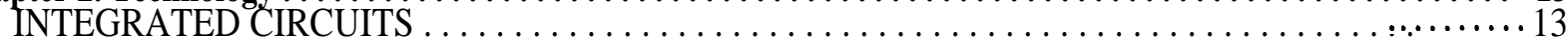

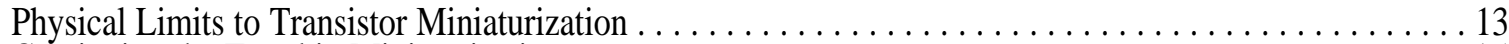

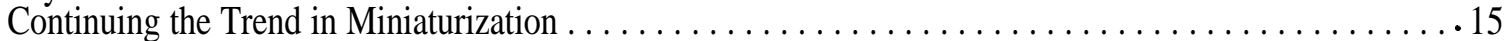

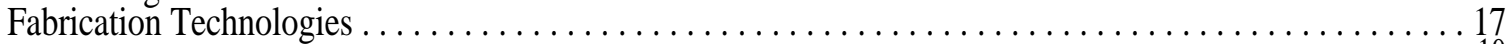

COMPUTING SYSTEMS TECHNOLOGIES $\ldots \ldots \ldots \ldots \ldots \ldots \ldots \ldots \ldots \ldots \ldots \ldots \ldots \ldots \ldots$

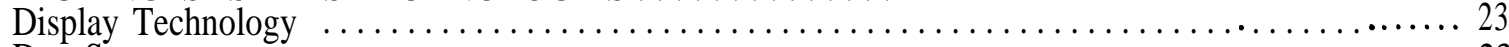

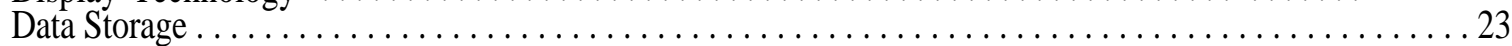

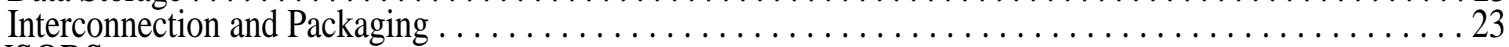

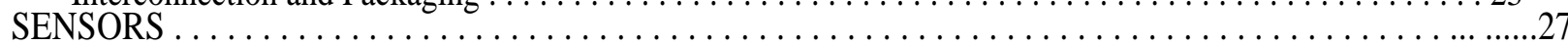

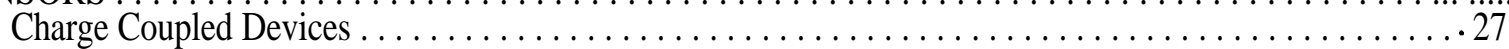

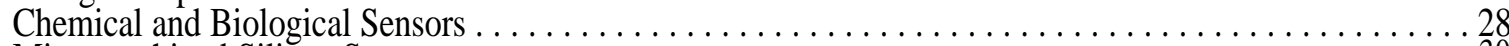

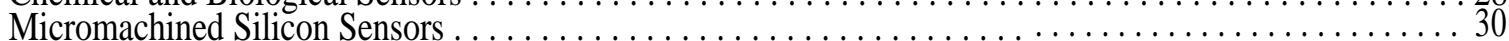

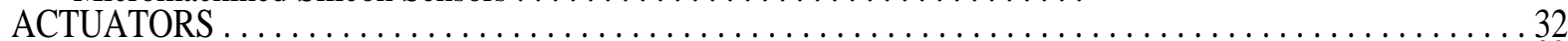

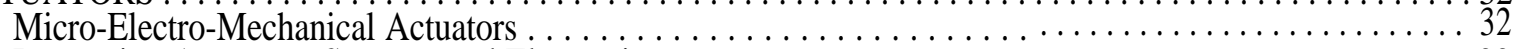

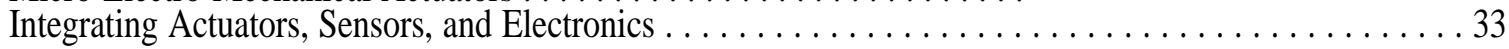

Appendix A. Fabrication Technology for Miniaturization $\ldots \ldots \ldots \ldots \ldots \ldots \ldots \ldots \ldots \ldots \ldots \ldots \ldots \ldots \ldots \ldots$

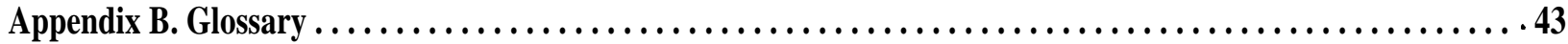

Boxes

Box

Page

2-A Molecular Machines ................................................. 20

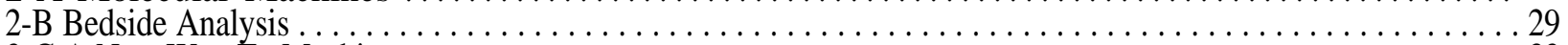

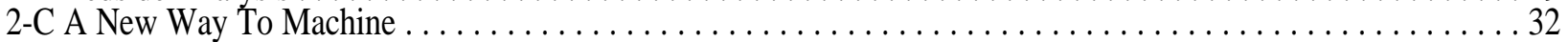

Figures

Figure

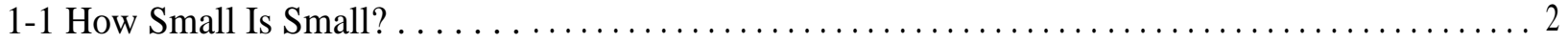

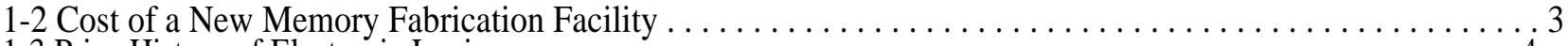

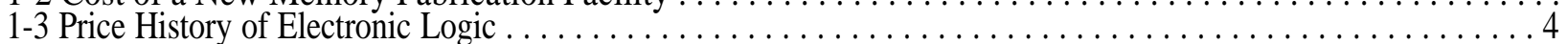

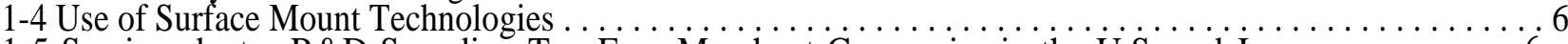

1-5 Semiconductor R\&D Spending-Top Four Merchant Companies in the U.S. and Japan $\ldots \ldots \ldots \ldots 6$

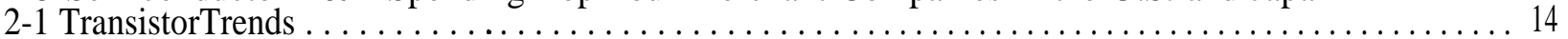

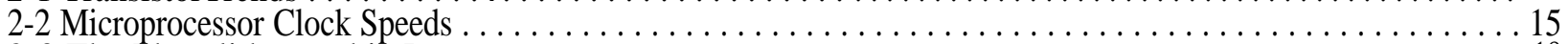

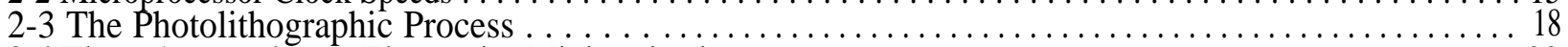

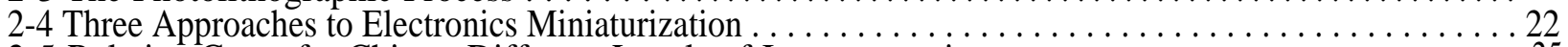

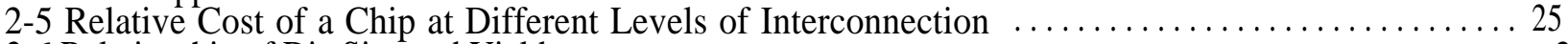

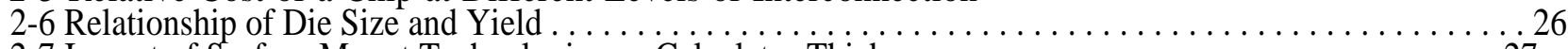

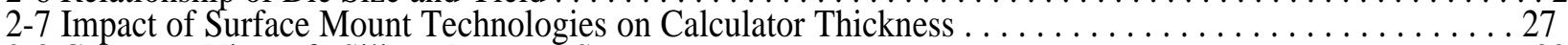

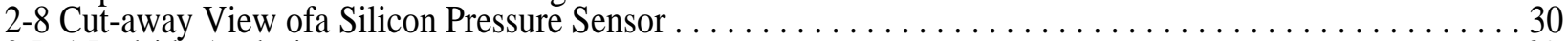

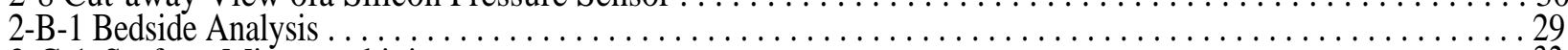

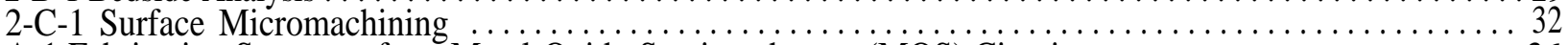

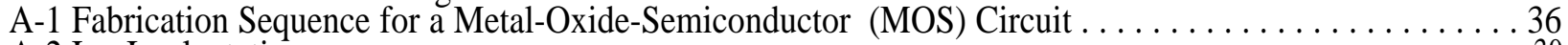

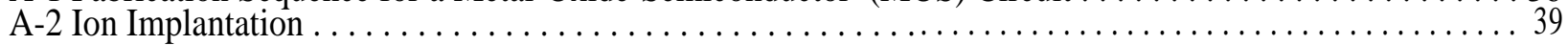


"Small is Beautiful." The truth of that statement is debated in economic and sociological circles, but when it comes to technology, there is no debate; small is beautiful because small is fast, small is cheap, and small is profitable. The revolution begun by electronics miniaturization during World War II is continuing to change the world and has spawned a revolution in miniaturized sensors and micromechanical devices.

Miniaturization plays a major role in the technical and economic rivalry between the United States and its competitors. It translates to market share and competitive advantage for many commercial and scientific products. Those companies and nations that can successfully develop and capitalize on miniaturization developments will reap handsome rewards. Personal computers, portable radios, and camcorders are examples of products that created massive new markets through miniaturization: they added billions of dollars to the GNP of countries where they were designed and built.

\section{FINDINGS}

The United States remains strong in miniaturization technologies research and development (R\&D), although the lead over other nations is less substantial than it has been in the past.

U.S. researchers continue to innovate and produce world-leading research despite strong research programs in Japan and Europe. There are some areas where Japanese or European research surpasses the United States in quantity and in a few cases in quality as well. But on the whole, U.S. researchers lead in miniaturization technology R\&D. The danger is that U.S. companies will lag other nations in implementing advanced technologies, especially when new technology is driven by a product or market dominated by another nation's industry.

The trends in silicon electronics miniaturization show no signs of slowing in the near future.

The current pace of miniaturization will produce memory chips (dynamic random access memory, DRAM) with a billion transistors and the capacity to store 1 billion bits (1 gigabit) of information around the year 2000. 'Transistors will continue to shrink until the smallest feature is around 0.1 micron (1 micrometer or one millionth of a meter). By comparison, today's most advanced mass-produced integrated circuits have features as small as 0.8 microns. A human hair is 50 to 100 microns in width (see figure 1-1). Achieving such tiny features will require a huge engineering and research effort. New fabrication equipment and processing techniques must be developed, pushing the cost of chip fabrication plants to over \$1 billion, compared to hundreds of millions for a current state-of-the-art plant (see figure 1-2). It is likely that despite the high costs, chips having features around 0.1 micron will be manufactured; progress beyond the era of O.1-micron transistors is uncertain.

The technology of semiconductor manufacturing is being applied to other fields to create new capabilities. Sensors created with semiconductor manufacturing technology hold the promise of widespread applications over the next 10 years.

Micromechanical sensors for pressure and acceleration have used semiconductor manufacturing technology for several decades now, but recent innovations allow further miniaturization, greater flexibility, and compatibility with microelectronics. A wider range of sensors can now be fabricated using micromechanical structures.

${ }^{1}$ This compares to today's most dense memory chips, which have about 4 million transistors and hold 4 million bits (4 megabits)of information. 
Figure 1-1 -How Small Is Small?

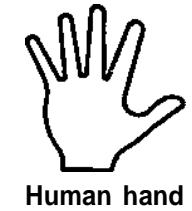

$\sim 0.1 \mathrm{~m}$ wide

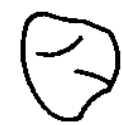

Grain of sand

$\sim$ t mm

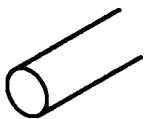

Human hair 50-100 microns wide

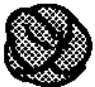

Smoke particle $\sim 4$ microns

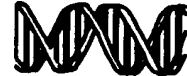

DNA $\sim 2 \mathrm{~nm}$ wide

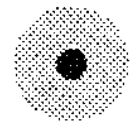

Atoms $1-4 \AA$

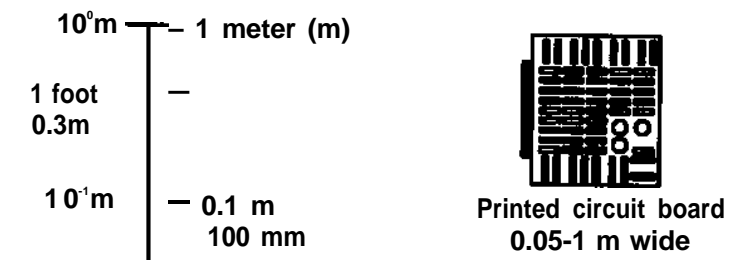

1 inch

$25.4 \mathrm{~mm}$

$10^{-2} \mathrm{~m}$.

$-0.01 \mathrm{~m}$

$10 \mathrm{~mm}$

1 centimeter
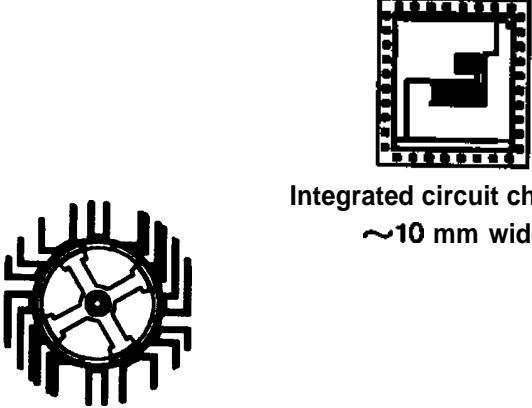

Micromechanical components

100 microns

10-1000 microns wide

Integrated circuit chip (die)

$\sim 10 \mathrm{~mm}$ wide

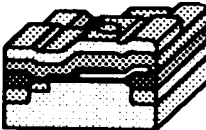

Transistor on integrated circuit 2-20 microns wide (smallest feature -0.8 micron)

1 microm
1 micron

$0.01 \mathrm{~mm}$

10 microns

$$
10^{-6} \mathrm{~m}-
$$

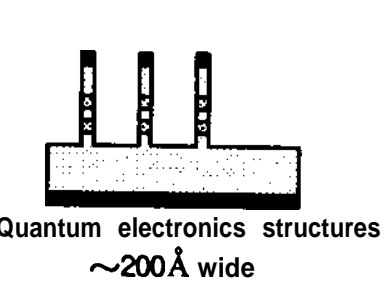

Quantum electronics structures $\sim 200 \AA$ wide

$0.01 \mathrm{~m}$
$10 \mathrm{~nm}$

$10 \mathrm{~A}$

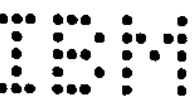

Atomic lettering using scanning tunneling microscope 65A high

Each increment on the vertical scale indicates change in scale bye factor of 10 . On the right of the scale are examples of miniaturization technologies. For comparison, several objects of similar scale are shown on the left.

SOURCES: Office of Technology Assessment, 1991. Data from Philip Morrison, Powers of Ten (San Francisco, CA: Scientific American Books, 1982); David Michael Freifelder, Molecular Biology (Portola Valley, CA: Jones and Bartett Publishers, 1987), p. 183; Douglas M. Considine, cd., Van Nostrand's Scientific Encyclopedia (New York, NY: Van Nostrand Reinhold Co., 1983). 
Figure 1-2-Coat of a New Memory Fabrication Facility (DRAM Fab)

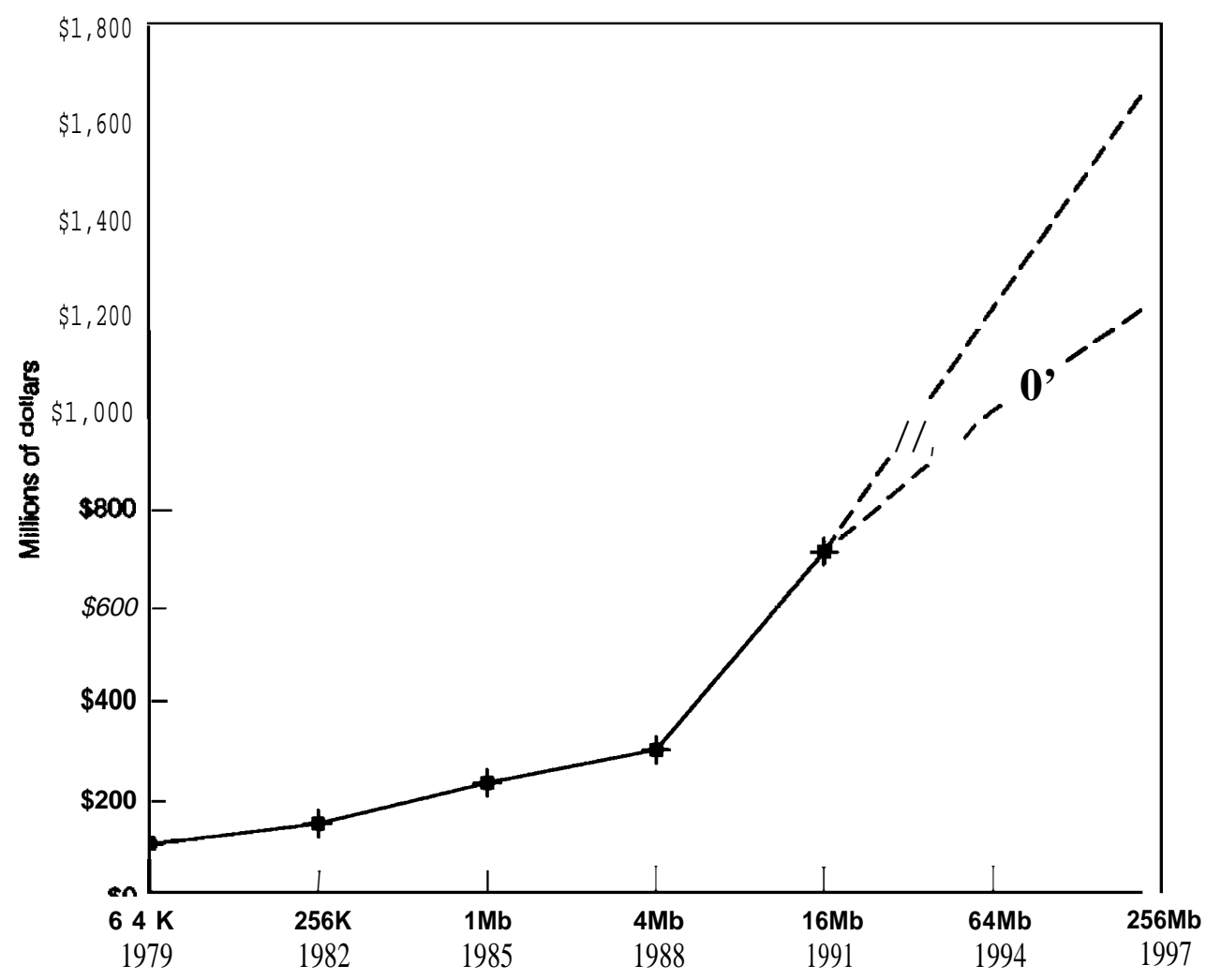

SOURCE: Dataquest (August 1991) and Graydon Laraboe, Texas Instruments, personal communication. Sept. 18, 1991.

Biosensors and chemical sensors that can sense gases and chemicals are being perfected using integrated circuit manufacturing techniques and will be used in medical, food processing, and chemical processing applications. The economics of microelectronics fabrication will result in these new sensors becoming cheap and ubiquitous since hundreds or thousands can be created on a single wafer. Integrating sensors with electronics promises to increase the versatility of sensors for consumer, medicine, automotive, aerospace, and robotics markets.

Materials and surface science research is critical to further advancement of all miniaturization technologies.

In every miniaturization technology-from silicon microelectronics to quantum electronics, to micromechanics and biosensors-better under- standing of materials and surface interactions will be a critical part of further technology advances. Better characterization of manufacturing processes will be necessary to make future generations of miniaturized semiconductors. Making practical miniaturized biosensors and chemical sensors will require better understanding of how to bond molecules to surfaces. Progress in micromechanics will depend on how well the mechanical and surface properties of materials like silicon are understood. Resolving problems in quantum electronics and molecular computing - the frontier of electronics miniaturization-are highly dependent on improved understanding and control of materials and surfaces. Basic research on material properties and surface interactions-especially in semiconductor processing and manufacturing-will be necessary for further miniaturization in many technology areas. 
Packaging is playing an increasingly larger role in miniaturization of electronics.

Trends in miniaturization are creating pressure in the electronics industry to improve packaging:

1. The proliferation of electronics into portable devices, consumer electronics, automobiles, and industrial applications is forcing more compact and ruggedized packaging;

2. The miniaturization of transistors causes them to operate faster and is forcing attention to better packaging because fast electronics must be packed close together to avoid delay in sending signals from chip to chip; and

3. As the costs of integrating more and more transistors onto the same piece of silicon increase, alternative ways to integrate transistors into a single package, such as multichip modules and surface mount technologies, are becoming more attractive.

\section{WHY IS MINIATURIZATION IMPORTANT?}

Miniaturization has inherent advantages, among them higher speed, lower cost, and greater density. Smaller electronics devices are generally faster because the signals do not have to travel as far within the device. Packing more functionality into a smaller or same-sized device reduces the cost of electronics. For example, a 1-megabit DRAM chip has four times the memory capacity of a 256-kilobit DRAM, but costs only about twice as much and occupies the same space as the lower capacity chip. Since the number of components (e.g., chips) on a circuit board largely determines the cost of the system, every 1-megabit DRAM used instead of four 256-kilobit DRAM reduces the number of memory chips on a board and reduces cost. Similarly, decreasing transistor size and greater integration has caused the price of logic devices to decline (see figure 1-3).

Miniaturization is important because it can create new markets by enabling new applications. Development of the microprocessor-a tiny com-

Figure 1-3-Price History of Electronic Logic

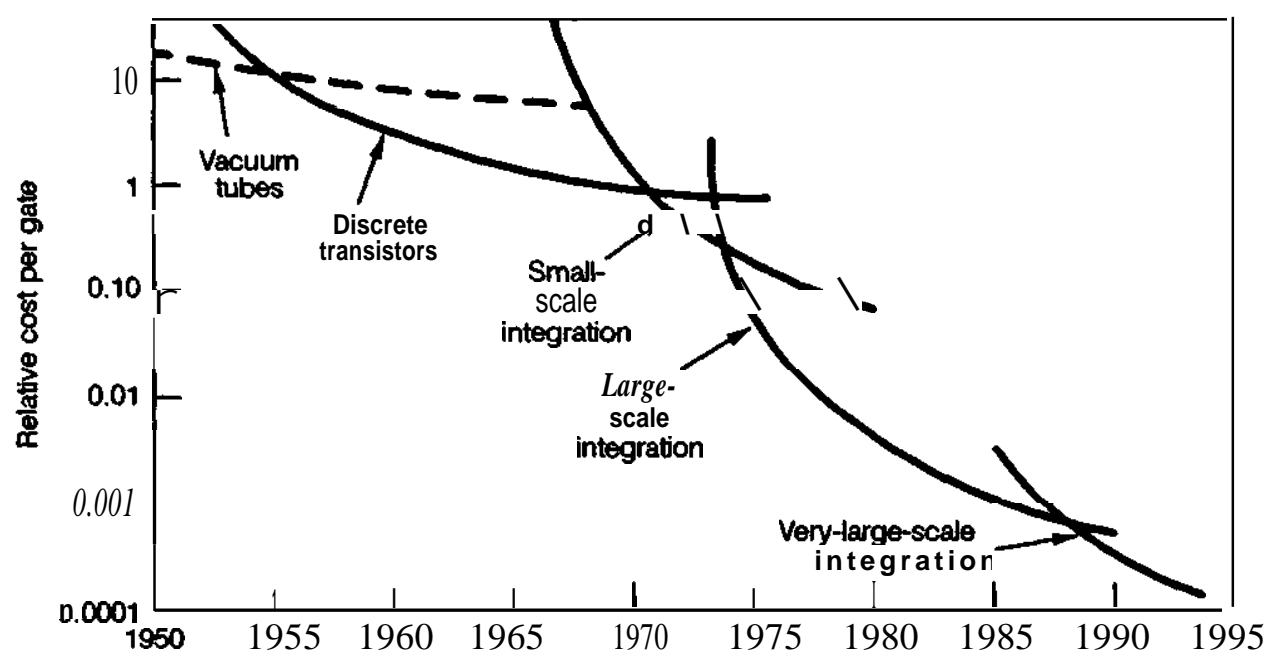

The price per gate-a circuit that performs a simple logic function -has continued on a steep downward trend since the introduction of the integrated circuit.

SOURCE: Adapted from John S. Mayo, "The Role of Microelectronics in Communications, "Microelectronics (SanFrancisco, CA: W.H. Freeman \& Co., 1977), p. 106. Copyright(c) 1977 Scientific American, Inc. All rights reserved. Additional data from Graydon Larrabee, Texas Instruments, personal communication, Sept. 30, 1991. 
puter on an integrated circuit-in 1970 led to a still expanding personal computer market currently valued at $\$ 70$ billion per year. Flat panel displays and improved chip packaging have led to battery-powered portable personal computers the size of notebooks (and some even smallerthe size of a checkbook), one of the newest markets created by miniaturization. Personal communications is a developing major consumer market created by miniaturization. With the simultaneous reduction in the size of personal computers and cellular telephones, the two are merging into a cordless appliance that can communicate with the rest of the world through a network. Nippon Electric Co. (NEC) already offers a laptop computer with a built in cellular phone for sale in Japan; similar products are under development by American companies.

\section{COMPETITIVENESS OF U.S. MINIATURIZATION TECHNOLOGIES}

The competitive position of U.S. R\&Din miniaturization technology remains strong, although competition from Japanese and European industry and governments has increased. As a result, the U.S. lead is less substantial than it was in the past. The rejuvenation of postwar Japanese and European economies has resulted in greater quality and quantity of research in those nations. There are now many more sources of competition in research worldwide.

Although U.S. R\&D strength is still sound, U.S. industry has a mixed record in the implementation of miniaturization technology. Many miniaturization technologies that are crucial to the success of consumer electronics, for example, were embraced by Japanese industry more quickly than by U.S. industry. Surface mount technology, which allows more electronic chips to be placed on a circuit board, has had greater penetration into Japanese industry than U.S. industry (see figure 1-4). Concerned with packing more electronics into portable consumer products, e.g., cameras, calculators, and stereo receivers, Japanese consumer electronics companies were eager to reduce the size of their products. U.S. companies that produce computers, industrial controls, and other large systems serve markets that are not as concerned with size and portability.

\section{Semiconductor}

Shrinking the size of transistors and their interconnections is being pursued vigorously by U.S. industry, government, and universities. The mainstream approaches to transistor miniaturization use silicon with designs similar to those of past devices. In many respects, the United States leads world research on smaller transistors. Design of miniaturized transistors draws heavily on basic sciences and computer modeling, areas in which the United States remains strong. Implementing smaller transistors in products, however, is a strength of Japanese industry. DRAM chips have historically been the first commercial products produced with each new generation of semiconductor manufacturing equipment. Since DRAM chips are made primarily by Japanese companies, they are the leaders in implementing small transistors in products.

$\mathrm{R} \& \mathrm{D}$ in semiconductor technology is done primarily by industry in both the United States and Japan. The Federal Government spends about $\$ 0.5$ billion per year on semiconductor R\&D. ${ }^{2}$ Japanese merchant semiconductor firms, companies that sell chips to other companies, have been outspending U.S. merchant firms on R\&D (see figure 1-5). Total U.S. and Japanese industry $\mathrm{R} \& \mathrm{D}$ spending is roughly equal; $\$ 3.7$ billion is spent in the United States and \$4 billion in Japan.

${ }^{2}$ Office of Management and Budget estimates for fiscal year 1990.

${ }^{3}$ Dataquest estimates for 1990. 
Figure 1-4-Use of Surface Mount Technologies

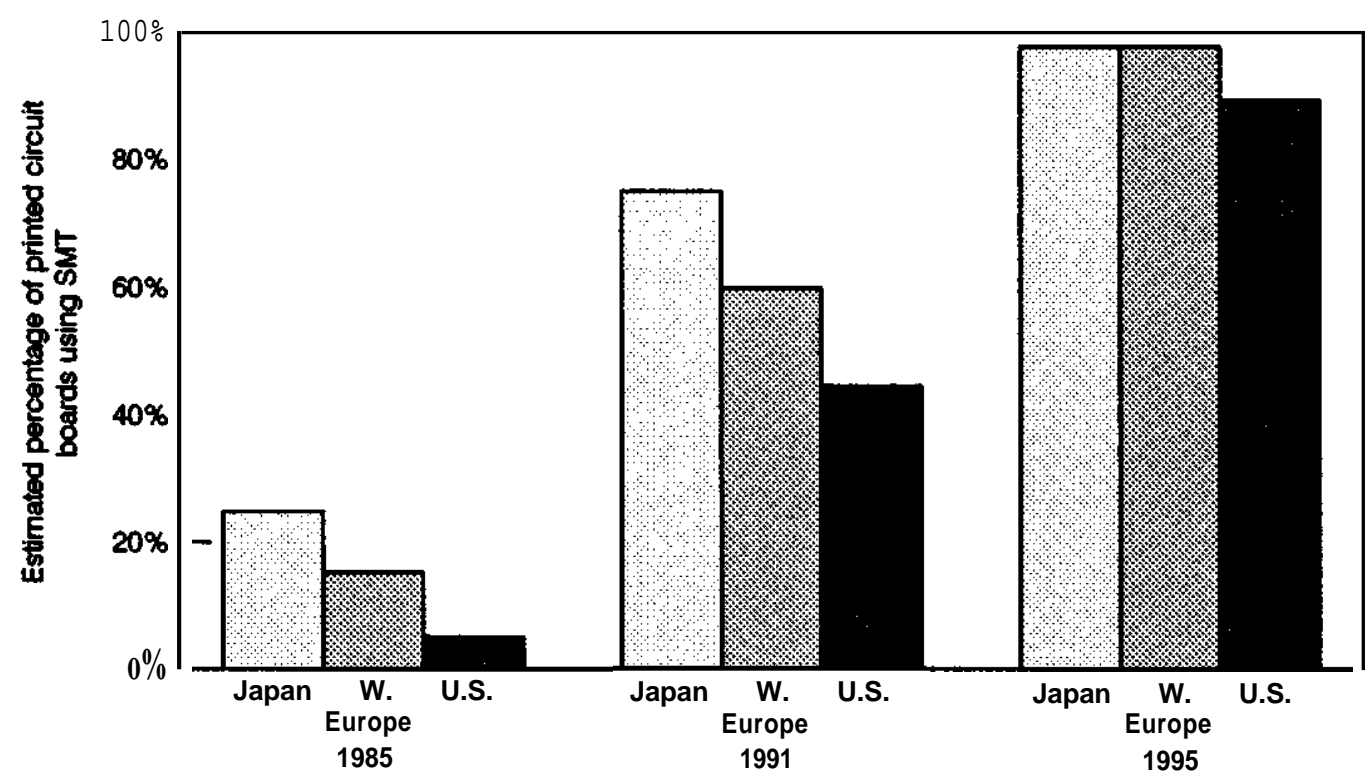

Use of surface mount technologies (SMT), which can reduce the thickness and volume of electronic products, has lagged in the United States compared to other nations. This graph shows past, current, and projected estimates of SMT use on printed circuit boards for Japan, Western Europe and the United States.

SOURCE: Office of Technology Assessment, 1991. Data from VLSI Research, Inc. (Fall 1991).

Figure 1-5-Semiconductor R\&D Spending-Top Four Merchant Companies in the United States and Japan

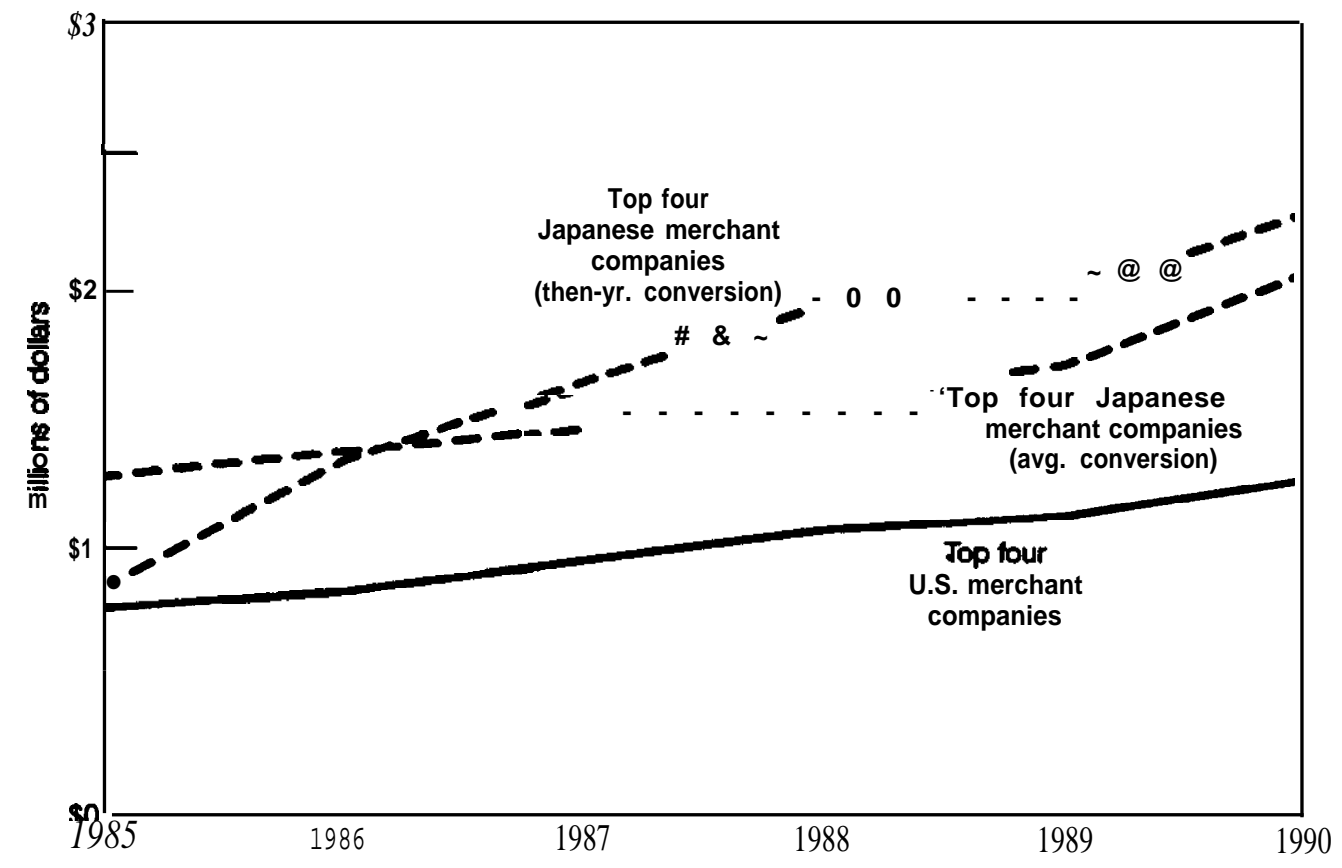

U.S. semiconductor merchant companies are outspent in R\&D by Japanese merchant firms. There are two curves for Japanese spending. The more dramatic increase results from converting yen to dollars at annual conversion rates. The other curve accounts for changes in the exchange rate by using a single conversion rate averaged over 5 years.

SOURCE: Office of Technology Assessment, 1991. Data from Dataquest. 


\section{Quantum Effect Devices}

Significant momentum is left in the current trend in silicon miniaturization. But what happens when today's silicon designs hit physical limits in further miniaturization, as many experts expect will happen in about 10 years? One option will be to change the way switching devices (used to make computer logic) are made. Several alternative approaches are being pursued by the United States, Japan, and Europe. One approach-quantum effect electronics-is still aresearch topic, but is receiving worldwide support. In the United States, about $\$ 8$ million are spent annually by the Federal Government in this area. Most of the research is being sponsored by the Defense Advanced Research Project Agency (DARPA), with significant efforts at other DoD research agencies and the National Science Foundation (NSF). Industry support for this basic research is sparse; AT\&T Bell Laboratories and Texas Instruments both have research activities in the area. IBM has a small research effort as well. Most of the research in the United States is being conducted at universities supported by government agencies.

The United States leads in research related to quantum effect devices, but this lead competes with other nations' efforts. Japanese efforts are significant and are beginning to make an impact; the Japanese involvement at recent conferences has grown. The United States can expect to maintain its lead in research, but, as the research moves toward development and implementation, the competition with Japanese researchers and corporations grows more fierce. Japanese industries have the advantage of being the world leaders in semiconductor processing technology and optoelectronics technology. ${ }^{4}$ U.S. companies have the advantage of proximity to the world's leading researchers in the field. The Japanese Government will spend less than $\$ 3$ million in fiscal year 1991 on quantum effect device research. With two new projects initiated in 1991 and 1992, funding will increase to about $\$ 6$ million per year over the next 5 years. Japanese industry is actively pursuing quantum effect device research. Fujitsu and NTT have the two largest research efforts. Estimates of Japanese industrial investment in the field are difficult to confirm, but the sum may be more than double the government investment. The Ministry of International Trade and Industry (MITI) 1992 Large Scale Program, "Angstrom Technologies," will also fund research in quantum electronics. The program will spend 25 billion yen (\$183 million) over ten years to conduct research on technologies at the scale of angstroms. It is not known, however, how much funding will go to quantum electronics.

\section{Molecular and Biological Computing}

One proposed way to continue the miniaturization of computers is to use individual molecules as switching devices in place of today's semiconductor transistors. U.S. investment in research related to molecule-based computing has diminished substantially since a period of intense interest in the early 1980s. NSF was the principal funding source for much of the original research. NSF's current funding for molecule-based computing is a few hundred thousand dollars and may end next year. Funding from U.S. industry is limited to a few venture capital firms and Digital Equipment Corp. Much of the effort in the United States has turned to development of molecular or biologically derived materials for applications in computing-related areas. For example, films of molecules rather than individual molecules $^{5}$ are being considered for use in optical disks and other data storage technologies.

Japanese and European governments continue to fund molecular computing research. However, differences in terminology complicate comparisons of programs between countries. Portions of one of the Japanese Exploratory Research for Advanced Technology (ERATO) projects are designed to pursue molecule-based com-

\footnotetext{
${ }^{4}$ Many current applications for superlattices-a structure used to create quantum effect devices-involve oPto electronics.

${ }^{5}$ Films of bacteriorhodopsin, a light sensitive biological molecule, are being prepared by researchers at Syracuse University, Mitsubishi, and a few American companies.
} 
puting $^{6}$ and the Ministry of International Trade and Industry (MITI) Basic Technologies for Future Industries Program spends about 300 million yen ( $\$ 4$ million) on research for biological molecular computing.

\section{Packaging and Interconnection}

As electronics become more ubiquitous, faster, and denser, the pressure on improving electronic packaging technology will increase. Federal funding has not addressed the issue of packaging as a whole, but many different programs fund research on packaging. Technologies such as surface mount technologies (SMT) and multichip module (MCM) technology can place more semiconductor chips into a system than traditional packaging. SMT is maturing and is seeing widespread application. Over 50 companies and research institutions worldwide are now pursuing MCM or related technology. ${ }^{7}$ One focus of the Microelectronics and Computing Technology Consortium (MCC)-a consortium of U.S. companies-is packaging and interconnection technology, including MCM technology. A National Research Council report in 199@ found that the American and Japanese industries are about equally matched in printed wiring boards, multichip modules, and other interconnection technology. The report also found that U.S. industry was dependent on Japanese suppliers for materials required for several packaging technologies.

\section{Biosensors and Chemical Sensors}

There is intense worldwide interest in developing biosensors and chemical sensors, particularly in Japan. Biosensors can detect the presence of chemicals or molecules such as glucose, urea, and oxygen. Diverse applications are seen in medical diagnosis, industrial process monitoring and control, fermentation process control, and food quality monitoring. Most of the $R \& D$ is being done by industry. Within the Federal Government, the Department of Defense (DOD) is a primary supporter of biosensor R\&D with the National Institutes of Health supporting some development.

According to a 1989 Japanese Technology and Evaluation Center (JTEC) Panel report, the U.S. efforts in chemical and biological sensors trail those of Japan. ${ }^{9}$ The panel report rated Japanese efforts more advanced in commercialization, product development and quantity of basic research. In quality of research, the U.S. and Japanese efforts were considered equal. OTA interviews in the biosensor industry indicate the situation has not changed much since the JTEC report. According to the report, in 1985 there were more Japanese publications and patents for biosensors than U.S. publications and patents. ${ }^{10}$ A consortia has been established by 35 Japanese companies to conduct R\&Din biological sensors. The annual budget for the consortia is about $\$ 2$ million from industry matched by funds from the Ministry of Agriculture, Forestry and Fisheries." In addition, a large number of Japanese companies are funding independent development of biosensors. ${ }^{12}$

\section{Micro-Mechanical Systems}

Development of new manufacturing techniques by researchers at American universities during the 1980s has led to an expansion of interest in micromechanics around the world. Build-

\footnotetext{
${ }^{6}$ Kunitake molecular architecture project (1987-92).

${ }^{7}$ Dennis Herrel and Hassan Hashemi, "Hybrid Wafer Scale Integration, “ MCC Technical Report P/I-329-89, 1989.

${ }^{8}$ National Research Council, Commission on Engineering and Technical Systems, National Materials Advisory Board, Materials for Hi@ Density Electronic Packaging and Interconnection (Washington, DC: National Materials Advisory Board, March 1990).

${ }^{9}$ U.S. Department of Commerce, “JTECH (Japanese Technology Evaluation Program) Panel Report on Advanced Sensors in Japan," January 1989, p. 184.

${ }^{10}$ Publications: 59 Japan, 35 United States; patents: 74 Japan, 9 United States.

${ }^{11}$ High Technology Business, September-October 1989, p. 29.

${ }^{12}$ National Science Foundation, Japanese Technology and Evaluation Center (JTEC), Viewgraphs for "JTEC Workshop on Bioprocess Engineering in Japan" (Washington, DC: National Science Foundation, May 21, 1991); and U.S. Congress, Office of Technology Assessment, Biotechnology in a Global Economy, OTA-BA-494 (Washington, DC: U.S. Government Printing Office, October 1991).
} 
ing upon established semiconductor processing techniques, researchers have been able to fabricate elaborate mechanical structures: motors and turbines as wide as a hair (see photograph), cantilevers capable of measuring acceleration, and gear assemblies smaller than a fleck of dust. These new techniques are closely related to techniques traditionally used to create mechanical structures for use as pressure sensors. The first applications with sizeable markets for the new technology are in sensors and instrumentation. Future applications may involve micromechanical actuators or systems of actuators and sensors. Some niche applications are already using micromechanical actuators made with conventional milling and extrusion techniques (non-microelectronics techniques).

Researchers at U.S. universities are the acknowledged leaders in micromechanical sensor research. The European nations, especially Germany, have a strong technology base in sensor technology and are supported with extensive government funding. Germany's Karlsruhe Nuclear Research Center and Fraunhofer Institute codeveloped a new lithographic process for making relatively thick (hundreds of microns) micromechanical structures, called LIGA. Although their industrial research in pressure and acceleration sensors is impressive, the Japanese trail the United States and Europe in R\&D.

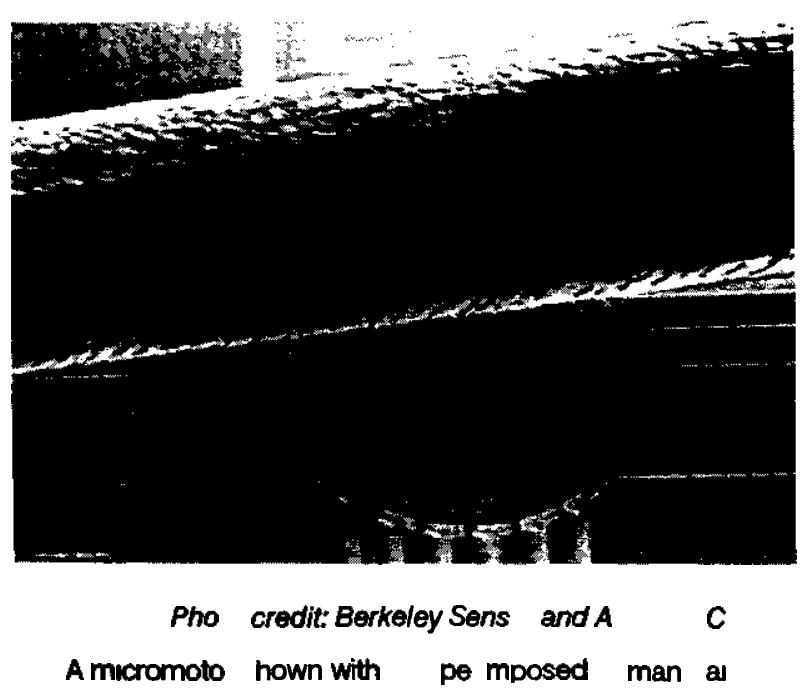

Although still relatively modest in scale, in the last few years funding for micromechanics has been increasing rapidly. In the United States, research in the field is sponsored by several different agencies, including DOD, NSF, the National Institutes of Health (NIH), the National Aeronautics and Space Administration (NASA), and the Department of Energy (DOE). Total Federal spending in the field is planned to be over $\$ 15$ million in 1991-from under \$6 million in 1990 and 1991. DOD and NSF were early supporters of the technology. DARPA has been spending about $\$ 2$ million per year, and plans to spend about $\$ 3$ million per year in 1992. NSF has been funding much of the research at universities since 1983 at about $\$ 2$ million per year. Efforts at the DOE have been mostly at Sandia and Lawrence Livermore National Laboratories. Starting in 1991, DOE will spend about $\$ 16$ million over 3 years at Louisiana Technical University (LTU) as directed by Congress. Other State governments including California and Louisiana have shown interest in promoting micromechanics research.

On the whole, U.S. industry is playing "wait and see" while trying to sort out what commercial applications the technology might have. Sensor and instrumentation companies, however, see clearer potential for applications and are pushing forward more aggressively. Analog Devices, Inc., for example, has developed the first commercial product that uses the new manufacturing technique-an acceleration sensor targeted for automobile airbag deployment applications. Other companies are abandoning micromechanics. A pioneer in the field, AT\&T Bell Laboratories, terminated its efforts in micromechanics. U.S. industry spent over \$20 million in fiscal year 1991 on micromechanics $R \& D$.

In Europe the largest and most advanced research efforts are being pursued by Germany. In 1990, the Germany Federal Ministry for Research and Technology initiated a 4-year program that will spend 400 million marks (\$230 million) on research. The German Government recently announced it will extend the program for at least another year. The Karlsruhe Nuclear Research Center and the Fraunhofer Institute are conduct- 
ing leading research in micromechanics. The Fraunhofer Institute is designed to encourage participation from industry and receives about 50 percent of its funding for micromechanics from industry. A consortium of German companies has licensed technology from Karlsruhe and is developing industrial applications based on the technology. Germany companies are interested in applications of the technology. Bosch has the largest effort, and Siemens and Messerschmidt also have significant R\&D efforts in the field.

Other European nations and industries are also pursuing micromechanics research. The most notable research efforts besides Germany are in Switzerland and the Netherlands. The Institute of Microelectronics in Neuchatel, Switzerland, is developing solid-state sensors and is active in the field of micromechanics research. The University of Twente and the University of Delft in the Netherlands are active in the field, with a new institute formed at the University of Twente-the Micro-Electromechanical Sensors and Actuators (MESA) Institute. Almost every nation in Europe has research activity in micromechanics - much of which is at universities.

Perceiving themselves as behind in micromechanics research, Japanese researchers are vigorously pursuing the technology with a new MITI program that will spend 25 billion yen ( $\$ 183$ million) over the next 10 years. The program, initiated in April of 1991, aims to develop microrobots for health and industrial applications. Micromechanical systems will be a major part of the program's research, although portions of the program will bean extension of a previous MITI program to develop miniature robots. Fiscal year 1991 funding for the program is only about $\$ 3$ million, but that will increase to over \$20 million annually as the program accelerates to full speed. Japanese industry typically adds its own investment of labor and equipment to work sponsored by MITI, so the total research effort associated with this program is substantially larger than the government investment. In addition to the MITI program, the Japanese Science and Technology
Agency (STA) funds research at several universities in Japan, including the University of Tokyo, ${ }^{13}$ at a total of about $\$ 1$ million per year. Japanese industry is pursuing research in the field, with 1991 expenditures estimated at over \$20 million. One of the largest industrial research efforts is at Toyota's central R\&D facility in Nagoya. Other industrial research is underway at NTT, NEC Corp., Ricoh Corp., IBM-Tokyo, and Matsushita Central Research. The MITI project has increased the interest of industry in micromechanics, and $R \& D$ can be expected to increase over the next several years.

\section{Fabrication Technology Research and Development}

Making miniaturized electronics, sensors, and micromechanics requires increasingly sophisticated manufacturing equipment as each generation of miniaturized components demands greater precision in fabrication.

Lithography-the technique used to etch features into integrated circuits-is one of the most challenging hurdles for future miniaturization of integrated circuits. The size of a transistor, the lines connecting transistors, and other devices in a circuit can only be made as small as the resolution of the tools used to make them. As the size of transistors become smaller, making tools of adequate resolution becomes increasingly difficult and more expensive. The current approach to increasing the resolution of lithography has been to reduce the wavelength of the light source, progressing from visible wavelengths, to ultraviolet, to deep ultraviolet. Now many experts are predicting that the trend will continue to x-ray lithography, but the outcome is far from certain. Some experts predict ultra-violet lithography will be useful in creating features as small as those possible with x-ray lithography. Using a technique called phase-shifting, this approach would require very sophisticated masks and computer software to be successful. Other lithographic ap-

\footnotetext{
${ }^{13}$ Others include Tohoku University, University of Osaka Prefecture, and Kyushu University.
} 
preaches such as electron-beam and ion-beam are considered viable options.

The debate surrounding $\mathrm{x}$-ray lithography has been well documented. ${ }^{14}$ The governments and industries of the United States, Japan, and European nations are all investing heavily in $R \& D$ for the next generation of lithography tools. Congress allocated \$60 million to DARPA in fiscal year 1991 to develop x-ray lithography technology, including research on mask development and laser $\mathrm{x}$-ray sources. There are a total of five synchro- trons currently in the United States for lithography research. Two more will come on line by 1993 in Baton Rouge, Louisiana and Upton, New York. Industrial support for $\mathrm{x}$-ray lithography using synchrotrons consists primarily of IBM and Motorola. AT\&T is focusing on $\mathrm{x}$-ray lithography using laser sources instead of synchrotrons. In Japan and Germany, government and industry are taking an aggressive approach to $\mathrm{x}$-ray lithography development. There are nine synchrotrons in Japan and two synchrotrons in Berlin for lithography research.

${ }^{14}$ For example, see Mark Crawford, "The Silicon Chip Race Advances Into X-rays," Science, Vo1. 246, Dec. 15, 1989, pp. 1382-1383;U.S. Congress, Congressional Budget Office, Using R\&D Consortia for Commercial Innovation: SEMATECH, X-ray Lithography, and High-Resolution Systems (Washington, DC: Congressional Budget Office, July 1990), pp. 69-87; U.S. Congress, Senate Committee on Commerce, Science, and Transportation, Subcommittee on Science, Technology, and Space, Semiconductors and the Electronics Industry, Serial No. 101-771, May 17, 1990; and John Markoff, "Etching the Chips of the Future," New York Times, June 20, 1990, p. D1. 


\section{INTEGRATED CIRCUITS}

Since World War II, system designers have tried to create smaller electronics. Reduction in the size of vacuum tubes progressed slowly as practical limits were encountered. Replacement of vacuum tubes with transistors in the 1960s was a breakthrough in miniaturization. Transistors became smaller, but each transistor was packaged individually, thus mounting large numbers of transistors on a circuit board still resulted in large assembly units. Invention of the integrated circuit overcame that problem by embedding many transistors on a single silicon chip that could be then connected to other components on a circuit board. Integrated circuits accelerated the drive toward transistor miniaturization because, unlike discrete transistors, the connections between transistors could shrink with the transistor. The smaller the transistor and its interconnections, the greater the transistor density (the number of transistors in a given chip area). As chip designers were able to pack more and more transistors onto a single silicon chip, the number of transistors per chip increased nearly a hundred fold each decade (see figure 2-1). As the size of a transistor shrinks it operates faster, leading to faster computation (see figure 2-2).

If this trend continues, sometime after 2000 the smallest feature on an integrated circuit will be about 0.1 micron ( 1 micron is 1 micrometer or one millionth of a meter). For comparison, today's dynamic random access memory (DRAM) transistor has a smallest feature of about 0.8 micron. The equivalent capacity of a DRAM ${ }^{1}$ chip will be over 1 billion bits (1 gigabit). With O.1-micron transistors, microprocessors will contain over 400 million transistors. Industry experts believe that such densities are possible and that there are no fundamental physical limits to prevent achieving them.

The time needed to overcoming engineering and manufacturing problems to reach these high densities is uncertain. Several problems must be overcome to develop high-density chips, including: 1) higher resistance of the minute connections between transistors and within transistors; 2) the tendency of very small transistors (about 0.1 micron) to "leak," rendering them useless; and 3) lack of high-volume manufacturing equipment capable of creating very small features.

Fabrication technologies, especially lithography, have paced the miniaturization of transistors in the past and may ultimately determine the practical economic limits of transistor miniaturization. Fabrication equipment used to make circuits at 0.1 microns must be affordable and capable of sustaining reliable, high-volume production. Electron-beam lithography can now produce features much smaller than 0.1 micron, but is unable to manufacture a sufficient volume of chips to be economical. ${ }^{2} \mathrm{X}$-ray lithography and phase-shifted masks (another lithography technique using visible light) are the two most likely contenders for achieving manufacturable O.1-micron transistors. Other prospects include projection electron beam, and projection focused ion beam.

\section{Physical Limits to Transistor Miniaturization}

What are the prospects for increasing densities of transistor based integrated circuits beyond 1 gigabit DRAM and 400 million transistor microprocessors? On this question, experts' opinions

\footnotetext{
${ }^{1} \mathrm{DRAM}$ is the primary $\mathrm{m}_{\text {ean }} \mathrm{s}$ of storing information in a computer system temporarily while the computer is working on the information. ${ }^{-}$Massstorage (e.g., hard disk) is used for permanent storage.

${ }^{2}$ Electron-beam lithography is currently used in production of masks and may be useful in some production applications, e.g., manufacturing chips for high-performance computers.
} 
Figure 2-1 -Transistor Trends

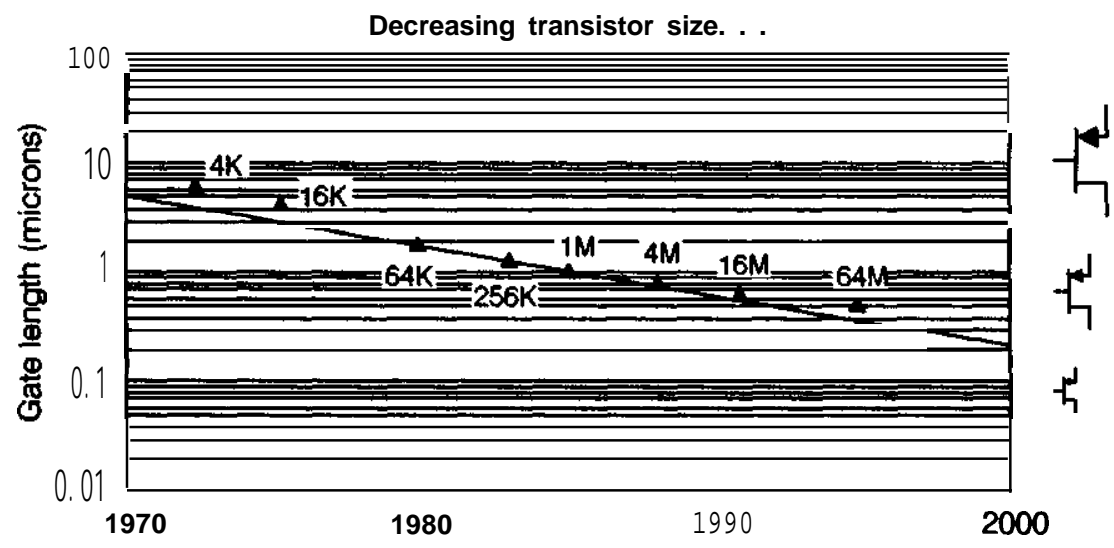

... and increasing die (chip) size. . .

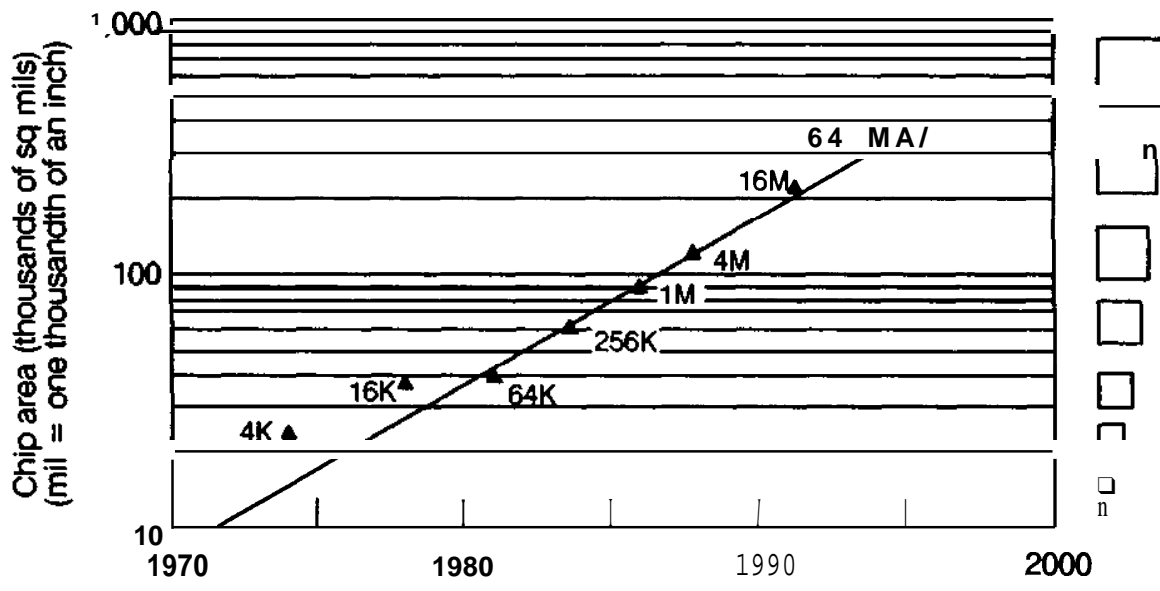

$\ldots$ is resulting in rapid growth in the number of transistors per chip.

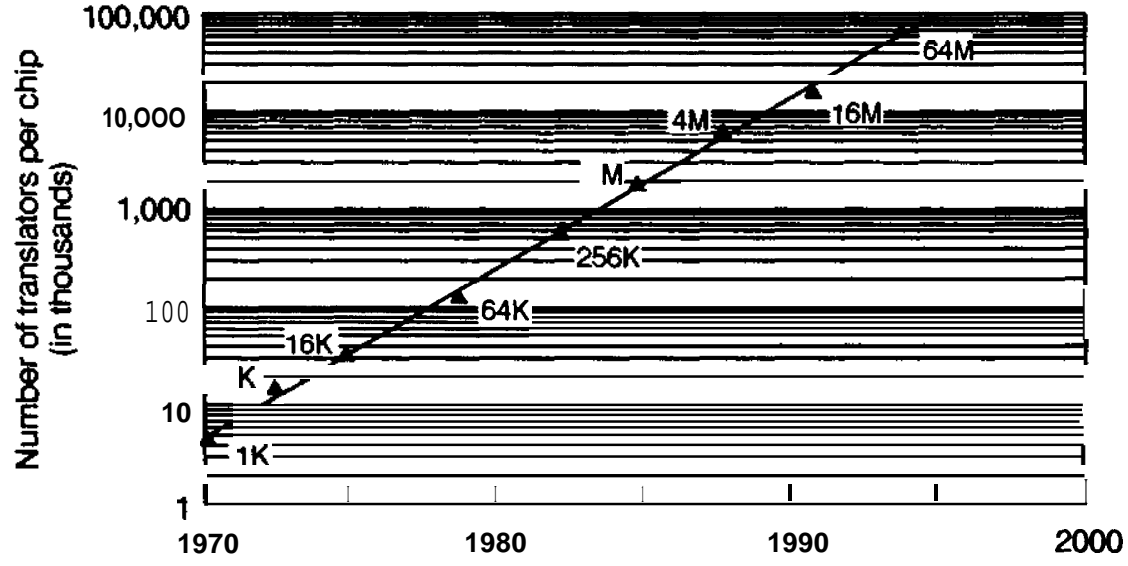

SOURCE: Office of Technology Assessment, 1991. Data from Intel Corp., David Ferry, Arizona State University, and Graydon Larrabee, Texas Instruments. 
Figure 2-3-The Photolithographic Process
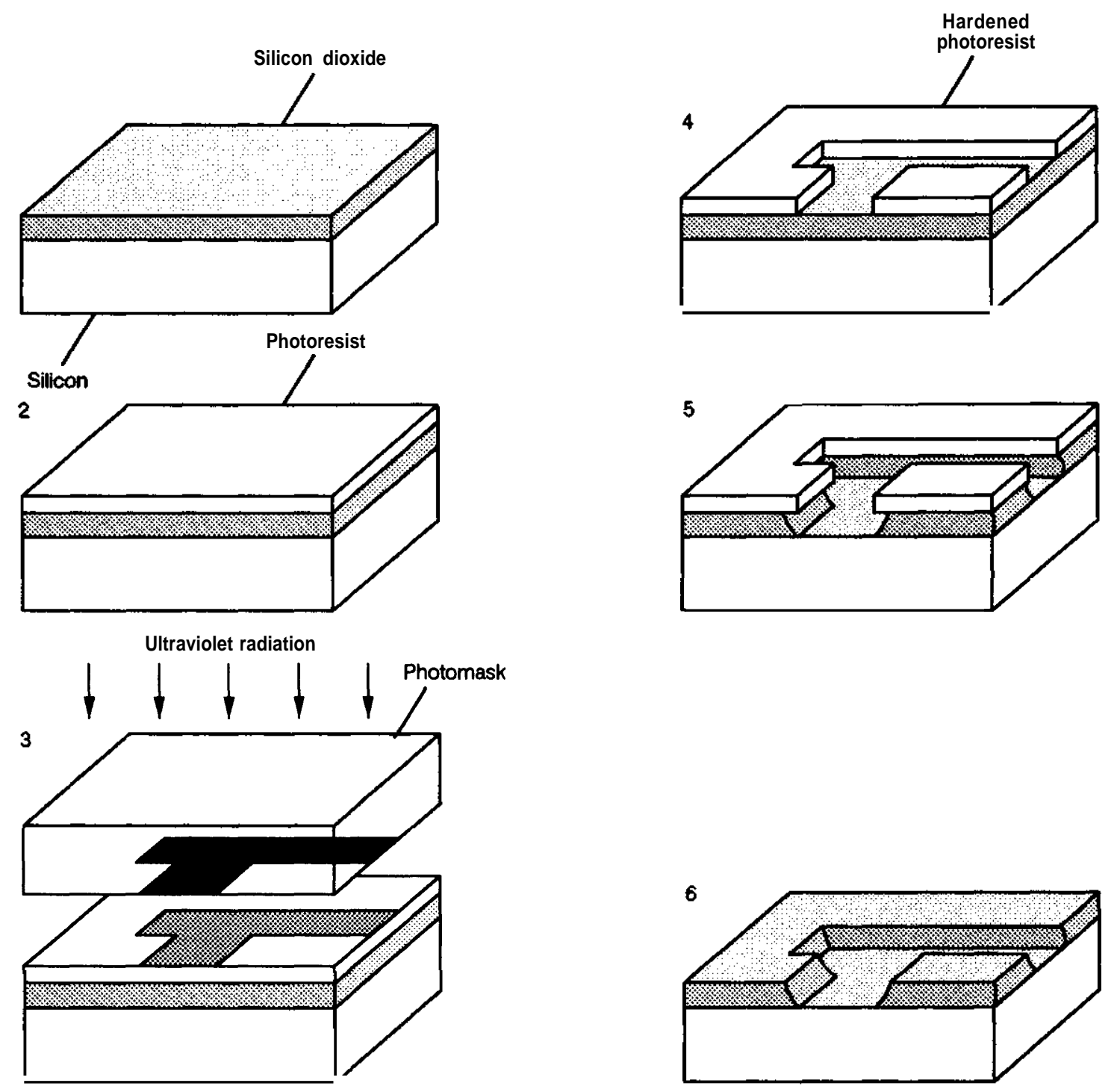

Photolithography is the process by which a microscopic pattern is transferred from a photomask to a layer of material in a circuit. In this illustration a pattern is shown being etched into a silicon dioxide layer (shaded) on the surface of a silicon wafer. The oxidized wafer (1) is first coated with a layer of a light-sensitive material called photoresist (2) and then exposed to ultraviolet light through the photomask (3). The exposure renders the photoresist insoluble in a developer solution; hence a pattern of the photoresist is left wherever the mask is opaque (4). The wafer is next immersed in a solution of hydrofluoric acid, which selectively attacks the silicon dioxide, leaving the photoresist pattern and the silicon substrate unaffected (5). In the final step the photoresist pattern is removed by means of another chemical treatment (6). There are variations on this process such as use of photoresists that become soluble instead of insoluble (4), and use of reactive gases instead of liquid acid solutions for etching (5).

SOURCE: Adapted from William G. Oldham, "The Fabrication of Microelectronic Circuits," Microelectronics (San Francisco, CA: W.H. Freeman \& Co., 1977), p. 47. Copyright (c) 1977 Scientific American Inc.-George V. Kelvin.

most common current technology uses visible or ultraviolet wavelengths of light for the photolithographic process.
The current state-of-the-art photolithography uses ultraviolet light, typically with a wavelength of less than 400 nanometers. Use of excimer laser 
sources could achieve wavelengths less than 200 nanometers. By improving mask technology, optics, and resists, ultraviolet systems may be usable to $\mathbf{0 . 2 5}$ - or 0.2-micron minimum feature size-dimensions that are expected to be needed in the mid-1990s. There are several lithography technologies that can create features smaller than 0.25 or 0.2 microns. The likely candidates for the next generation of lithography tools are x-ray lithographies, optical lithographies using phase shift masks, electron-beam lithographies, and ion-beam lithographies. Combinations of the techniques are also possible. (For a more detailed description of the photolithographic process, see app. A.)

Materials and the principles of surface sciences are fundamental to advancing miniaturization of transistors and electronics. Improvements in semiconductor manufacturing rely on understanding how materials react to processing and fabrication techniques. Physical phenomena underlying the critical fabrication steps are not well understood; knowledge has developed mostly from experience, not derivation from physical laws. Better understanding of the physical laws affecting small-scale structures would accelerate advances in miniaturization.

There are more speculative ideas for future fabrication technologies that would require even more rigorous understanding of surface interactions. Some exploratory work is currently underway to use proximal probes to fabricate integrated circuits. Using a scanning tunneling microscope (STM) or some variation of an STM, scientists are beginning to fashion crude structures from individual atoms and clusters of atoms. Researchers have been able to move or deposit atoms on or below surfaces to "draw" figures, maps, and company logos (see photograph). Even if these techniques do not result in usable manufacturing tools, they will increase our understanding of surface interactions of atoms, leading to better understanding of traditional semiconductor manufacturing. Even more speculative is the prospect of creating molecular-sized robots and machines that could be programmed

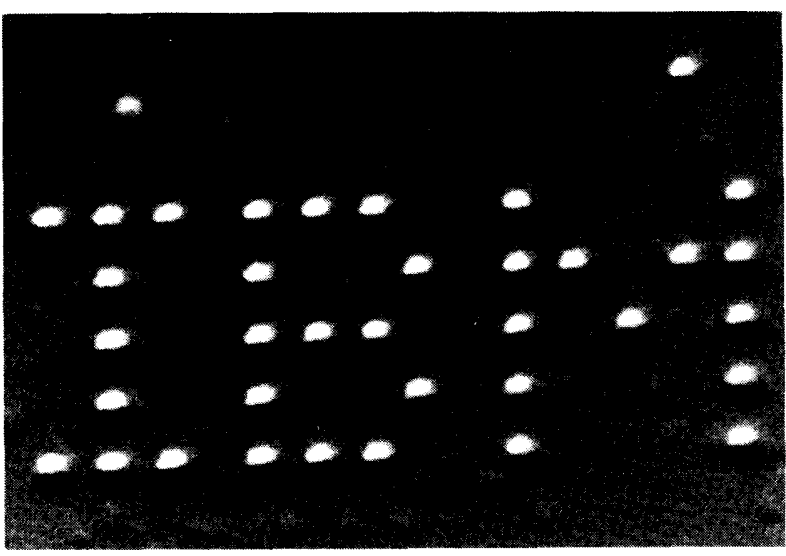

Photo credit: IBM

Scientists at IBM's Almaden Research Center used a scanning tunneling microscope (STM) to move xenon atoms around on a nickel surface and spell out the company name. The distance between each atom of xenon is about 13 angstroms.

to manufacture virtually any molecular structure (see box 2-A).

\section{COMPUTING SYSTEMS TECHNOLOGIES}

Computer systems have shrunk dramatically during the last 30 years. Mainframe computers once filled large rooms and required air conditioning to dissipate the heat generated by vacuum tubes and early transistors. Computers have become so small that laptops and notebook computers are more powerful than mainframe computers of 10 years ago. Equivalent power will soon be available in a checkbook-size package. Past miniaturization of electronics has played the major role in bringing this capability about and future miniaturization advances will be driven primarily by electronics (see figure 2-4). Other components in addition to integrated circuits also had to shrink in size to accommodate today's notebook-sized computers. Increased mass memory density, greater circuit board density, and thinner flat panel displays have improved computer system performance and allowed downsizing. Trends toward smallness are expected to continue as customers demand higher quality, higher performance, and portability from smaller boxes. 
The technical problems associated with making practical memory or logic systems from molecular devices are substantial and are not likely to be solved in this decade. The most vexing problem is the interface (connection) between the molecule and the circuit itself, a problem common to many forms of miniature transistors. At $0.1 \mathrm{mi}-$ cron, transistors begin to experience problems with high resistance, but a molecular transistor would be smaller still -0.001 microns or less. In order to avoid the electrical interface problem, some researchers are exploring optical interfaces. The diameter of an optical interface, however, is limited by the wavelength of light. The stability of the molecules is also a problem. To be useful for computation, a molecule must remain in a specific configuration until changed by an external signal. Individual molecules sometimes change configurations unexpectedly. Using clusters of molecules makes them easier to connect to circuits and reduces the errors in the stored data, but makes the computing device larger.

Related research is underway in the use of biologically derived or organic material for storing information or computing. These approaches minimize the interface and stability problems of individual molecule computation. A few U.S. companies are working on organic materials for optical disks and at least one Japanese firm, Mitsubishi, is working on an optical disk that will use bacteriorhodopsin as the storage material. ${ }^{5} \mathrm{Few}$ materials have suitable properties for computing; this has been a major stumbling block for molecular computing. U.S. research is directed at better understanding materials that might serve as the basis of future molecule-based computers. The more immediate results may be faster data storage, but proponents of molecule-based computing hope that the experience gained will lead to true molecule-based computing and processing systems in the future.

\section{Fabrication Technologies}

There are several manufacturing technologies that are important in making semiconductor devices. The most critical manufacturing technology by many accounts, is lithography-the techniques used to pattern and etch transistors and their interconnections on a substrate like silicon or GaAs.

There are several different lithographic technologies including photolithography, electronbeam lithography, ion-beam lithography, and xray lithography. In addition, there are several novel technologies still in early stages of development-e.g., scanned photolithography, combined patterning and growth, and proximal probe fabrication.

One of the most critical steps in lithography is exposing the resist-usually an organic compound layered on top of the semiconductor wafer-to an energy source (see figure 2-3). The energy source can be optical, ultraviolet, or x-ray. It can also be a beam of charged electrons or a beam of charged atoms (ions). Some of the resist is exposed to the energy source and some is not, depending on whether the resist lies under a transparent or opaque portion of the mask. In the places exposed to the energy source, the resist is modified so that certain chemicals can dissolve it. Exposing the wafer with a series of etching chemicals removes the resist and a layer of material (usually silicon dioxide) underneath. The remaining resist is then removed by another chemical that does not affect the semiconductor material. A layer of material is typically deposited again and the process is repeated-as many as 12 or more times-to make an integrated circuit. The minimum feature size that can be formed is determined primarily by the precision that the energy source can be focused to discriminate between the areas of the resist that are exposed to the energy and those that are not. ${ }^{7}$ The

\footnotetext{
${ }^{5}$ Robert Birge, Syracuse University, personal communication, July 29, 1991.

${ }^{6}$ There are different types of resists. Exposure to radiation causes some types to become susceptible to the subsequent etching step, and causes others to become resistant to etching.

${ }^{7}$ Other key factors are the ability to align subsequent layers of masks to create the proper vertical geometry and the ability to control the rate and direction of etching.
} 
Figure 2-3-The Photolithographic Process
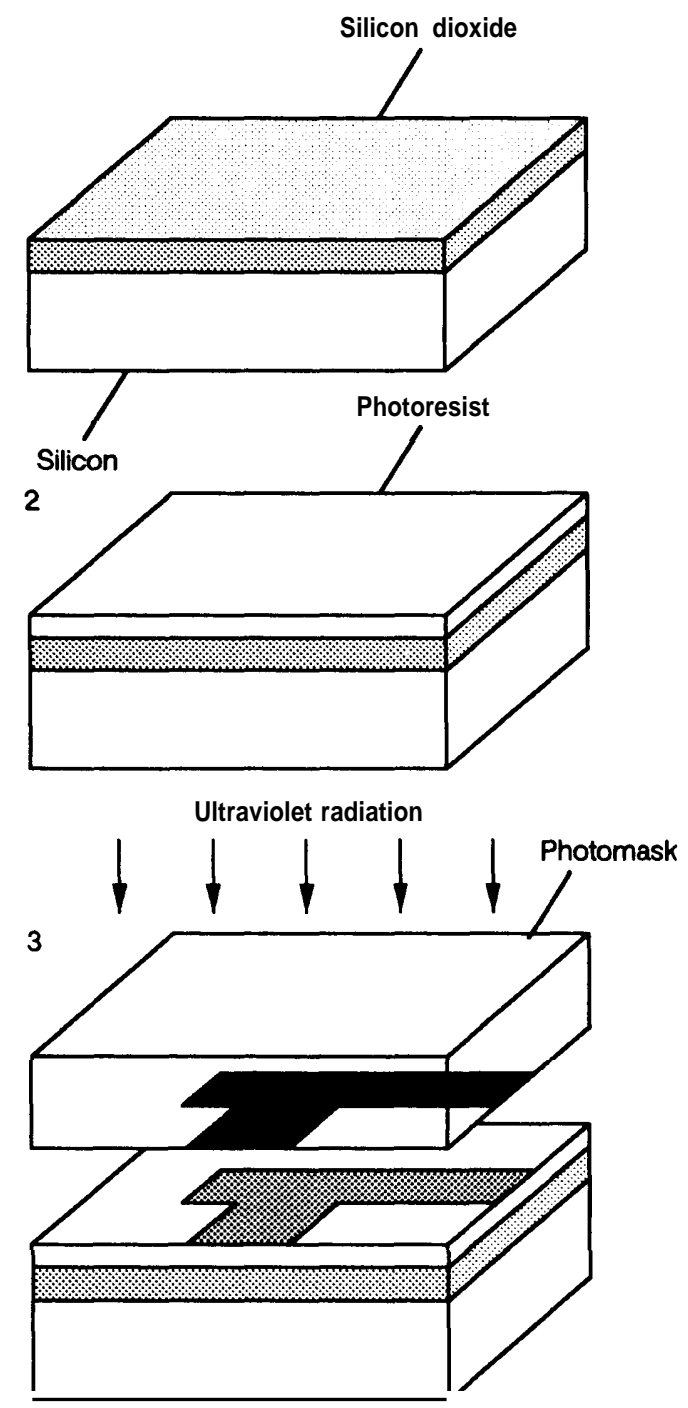
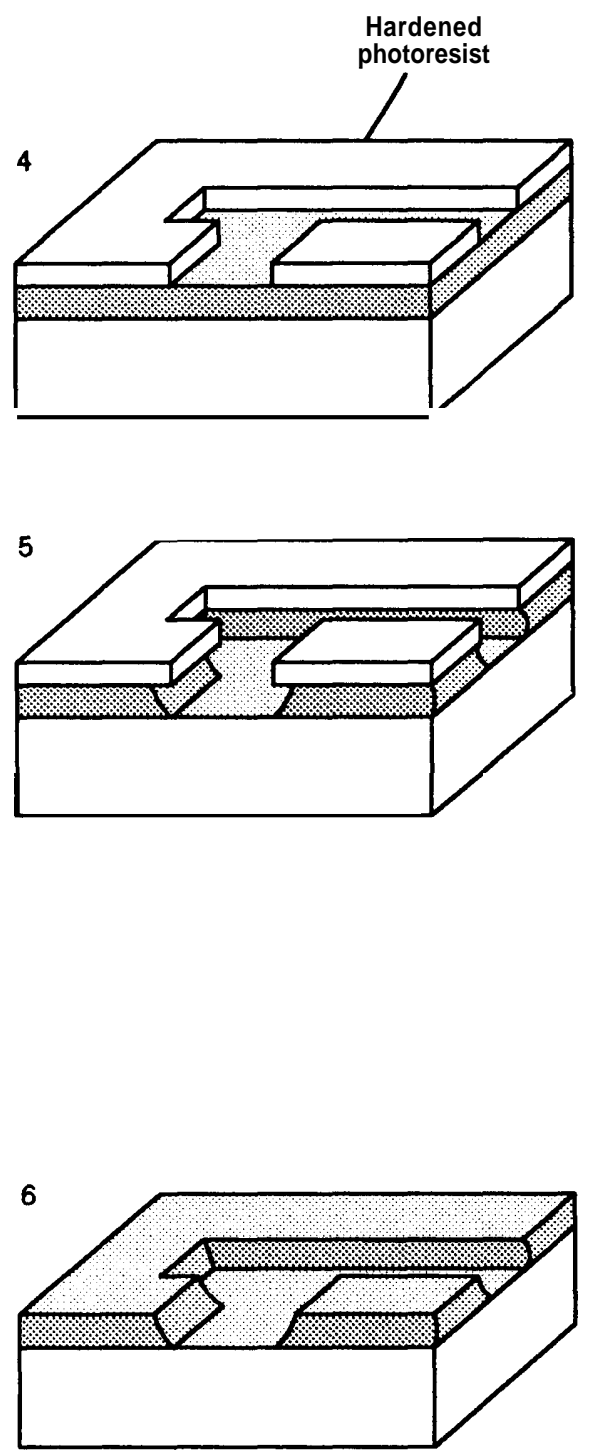

Photolithography is the process by which a microscopic pattern is transferred from a photomask to a layer of material in a circuit. In this illustration a pattern is shown being etched into a silicon dioxide layer (shaded) on the surface of a silicon wafer. The oxidized wafer (1) is first coated with a layer of a light-sensitive material called photoresist (2) and then exposed to ultraviolet light through the photomask (3). The exposure renders the photoresist insoluble in a developer solution; hence a pattern of the photoresist is left wherever the mask is opaque (4). The wafer is next immersed in a solution of hydrofluoric acid, which selectively attacks the silicon dioxide, leaving the photoresist pattern and the silicon substrate unaffected (5). In the final step the photoresist pattern is removed by means of another chemical treatment (6). There are variations on this process such as use of photoresists that become soluble instead of insoluble (4), and use of reactive gases instead of liquid acid solutions for etching (5).

SOURCE: Adapted from William G. Oldham, "The Fabrication of Microelectronic Circuits," Microe/ectronics (San Francisco, CA: W.H. Freeman \& Co., 1977), p. 47. Copyright (c) 1977 Scientific American Inc.-George V. Kelvin.

most common current technology uses visible or ultraviolet wavelengths of light for the photolithographic process.
The current state-of-the-art photolithography uses ultraviolet light, typically with a wavelength of less than 400 nanometers. Use of excimer laser 
sources could achieve wavelengths less than 200 nanometers. By improving mask technology, optics, and resists, ultraviolet systems may be usable to $\mathbf{0 . 2 5}$ - or 0.2-micron minimum feature size-dimensions that are expected to be needed in the mid-1990s. There are several lithography technologies that can create features smaller than 0.25 or 0.2 microns. The likely candidates for the next generation of lithography tools are x-ray lithographies, optical lithographies using phase shift masks, electron-beam lithographies, and ion-beam lithographies. Combinations of the techniques are also possible. (For a more detailed description of the photolithographic process, see app. A.)

Materials and the principles of surface sciences are fundamental to advancing miniaturization of transistors and electronics. Improvements in semiconductor manufacturing rely on understanding how materials react to processing and fabrication techniques. Physical phenomena underlying the critical fabrication steps are not well understood; knowledge has developed mostly from experience, not derivation from physical laws. Better understanding of the physical laws affecting small-scale structures would accelerate advances in miniaturization.

There are more speculative ideas for future fabrication technologies that would require even more rigorous understanding of surface interactions. Some exploratory work is currently underway to use proximal probes to fabricate integrated circuits. Using a scanning tunneling microscope (STM) or some variation of an STM, scientists are beginning to fashion crude structures from individual atoms and clusters of atoms. Researchers have been able to move or deposit atoms on or below surfaces to "draw" figures, maps, and company logos (see photograph). Even if these techniques do not result in usable manufacturing tools, they will increase our understanding of surface interactions of atoms, leading to better understanding of traditional semiconductor manufacturing. Even more speculative is the prospect of creating molecular-sized robots and machines that could be programmed

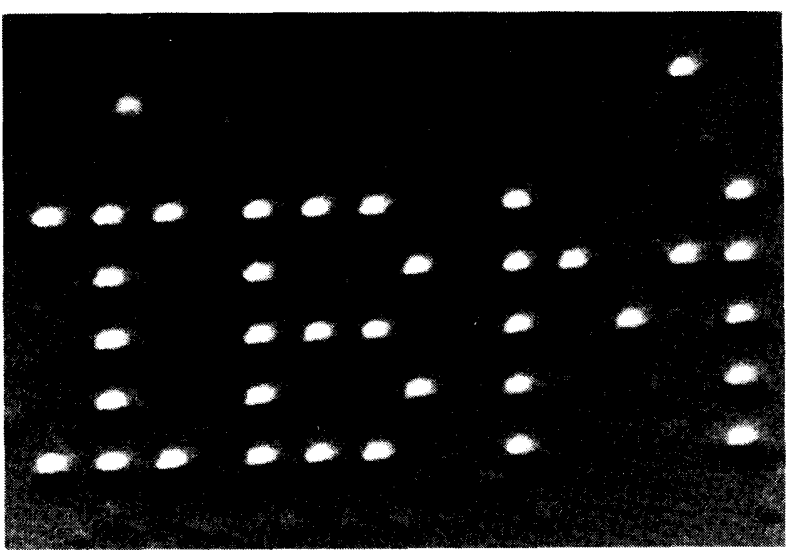

Photo credit: IBM

Scientists at IBM's Almaden Research Center used a scanning tunneling microscope (STM) to move xenon atoms around on a nickel surface and spell out the company name. The distance between each atom of xenon is about 13 angstroms.

to manufacture virtually any molecular structure (see box 2-A).

\section{COMPUTING SYSTEMS TECHNOLOGIES}

Computer systems have shrunk dramatically during the last 30 years. Mainframe computers once filled large rooms and required air conditioning to dissipate the heat generated by vacuum tubes and early transistors. Computers have become so small that laptops and notebook computers are more powerful than mainframe computers of 10 years ago. Equivalent power will soon be available in a checkbook-size package. Past miniaturization of electronics has played the major role in bringing this capability about and future miniaturization advances will be driven primarily by electronics (see figure 2-4). Other components in addition to integrated circuits also had to shrink in size to accommodate today's notebook-sized computers. Increased mass memory density, greater circuit board density, and thinner flat panel displays have improved computer system performance and allowed downsizing. Trends toward smallness are expected to continue as customers demand higher quality, higher performance, and portability from smaller boxes. 


\section{Box 2-A-Molecular Machines}

With electronics continuing to shrink and advances in the ability to make tiny mechanical devices, one wonders if there is a limit to the ability to manipulate small structures. Some theorists speculate that humans could control individual molecules precisely enough to build molecule-sized machines and robots. These machines would be so small that millions would fit in one of the micromotors described elsewhere in this report. Various terms have been used to describe the concepts; ${ }^{1}$ here they will be referred to as "molecular machines."

The process of DNA replication and protein generation demonstrates that molecules can store information and use it to fabricate complex molecular structures. Molecular machine theorists claim humans will be able to create molecular machines to perform similar functions. ${ }^{2}$ These molecular robots could be programmed to manufacture virtually any molecule-based structure Possible--everything from hamburgers to spaceships. According to proponents, such technology could control pollutants, create flawless materials, and provide almost limitless computing power. But they warn that there are potentially dangerous applications such as weapons more powerful than nuclear bombs, or machines that replicate uncontrollably, reducing the earth to a "gray goo."

Significant barriers prevent the immediate implementation of molecular machine concepts. The only tools that can directly manipulate molecules are proximal probes, e.g. the scanning tunneling microscope (STM). Proximal probes allow imaging and manipulation of atoms on a surface. Although STM technology is advancing rapidly, manipulating molecules and atoms is awkward and time consuming-a simple molecule has yet to be fabricated. ${ }^{3}$ Problems with reliability of molecular-sized systems might make them impractical or delay their development. Because of their extremely small size, molecular machines are especially susceptible to influences such as thermal noise and radiation damage. Molecular machine system designers must compensate for these damaging effects. In many molecular machine application concepts, e.g., random access memory (RAM) or gene sequencing, molecular scale systems must interface with larger scale systems. In addition, molecular machines must interface with the outside world in order to be "programmed." The problems of interfacing molecules to larger scale systems hamper the ability to miniaturize many devices, ${ }^{4}$ including many of those proposed by molecular machine designers.

\section{Should Policymakers Be Concerned?}

Because of the tremendous impact molecular machine technology might have, there are calls for government to monitor progress in the field and fund research toward its realization. ${ }^{5}$ OTA attempted to determine the importance of molecular machine ideas to policymakers by assessing the basis of the molecular machine concepts and prospects for their development.

1Other terms used currently and in the past include "nanotechnology," "Feynman machines," "eutaxic control," "assemblers," and "nanobots." The term "nanotechnology" is particularly confusing because it is also used to refer to sub-micron electronics, micro-sensors, quantum electronics, and micromechanics. As a result, molecular machine concepts are often portrayed in the same light as those other technologies that are very different in size, technology base, and time to realization.

${ }^{2}$ For more detailed descriptions of molecular machine concepts, see K.E. Drexler, Engines of Creation (New York, NY: Anchor Books, 1986) or references 7 and 8.

3Donald Eigler, IBM Almaden Research Center, personal communication, May 1991. Fabrication of a simple molecule a reasonable near-term expectation.

${ }^{4} \mathrm{See}$ the descriptions Of transistor miniaturization in this chapter for details.

${ }^{5}$ See Susan G. Hadden et al., Assessing Molecular and Atomic Scale Technologies (MAST) (Austin, TX: University of Texas at Austin Board of Regents, 1989); and Chris Peterson, "Molecular Manufacturing for Space," Forsight Update, No. 12, p. 8. 
Despite the skepticism of many researchers contacted by OTAand the evident controversial nature of their ideas, there has been little written criticism of molecular machine Concepts. ${ }^{6}$ The two seminal articles on the topic of molecular machines were written by Richard Feynman ${ }^{7}$ and Eric Drexler. ${ }^{8}$ The arguments for and against molecular machines tend to be conceptual and not refined to the point of discussing architectures or systems the debate so far seems to center on whether such devices are possible within bounds of natural law. Written criticism tends to focus on more specific suggestions and architectures in the related field of molecule-based electronics rather than molecular machine concepts. The scarcity of criticism maybe due to the reluctance of scientists to denounce new concepts in publications.'

There are many concepts of what impossible within the bounds of natural law--e.g., steam-powered computers and interstellar travel-but they do not exist as technologies. While science can determine whether a concept is feasible, technology development is influenced by unpredictable economic and social factors. As a technology, molecular machines are non-existent; the only work to date has been conceptual and computational modeling.

When will molecular machine technology be developed? Estimates from the proponents of the concepts are 10 to 30 years, while others predict from centuries to "never." One of the basic components of a molecular machine technology base would be a protoassembler, a molecular machine capable of fabricating other molecular machines, The earliest prediction for development of protoassemblers is 5 to 10 years. ${ }^{10}$

\section{Is There a Government Role?}

To date, no proposal for research on molecular machine development has been received by a Federal agency. Basic scientific and engineering research in the fields of materials science, chemistry, molecular biology, advanced electronics, molecular modeling, and surface science are being funded by many Federal agencies and would be necessary precursors to the realizationof molecular machines. It is impossible to estimate the level of funding, however, since there is no exact definition of precursor technologies. There are a few small research efforts explicitly addressing molecular machines concepts in U.S. academia and industry and Japanese government."

Development of a framework for government regulation and oversight of molecular machine technologies has been suggested by several analysts, driven by fears of abuse or accidents associated with the development of these technologies. The communities of researchers working on these precursor technologies is rather small and the concern over accidents or misuse of the technology is well known among them. Government regulation at this stage would be premature, might hamper emerging research efforts, and have uncertain advantages. The question of regulation and oversight should be revisited and analyzed in greater depth if developments in the field bring the technology closer to reality. The development of the first protoassembler might bean appropriate milestone to reconsider government regulatory involvement.

6TA conducted a literature search of articles that reference the two articles and analyzed them for critical and supporting arguments.

${ }^{7}$ R. Feynman, "There's Plenty of Room at the Bottom," Miniaturization, A. Gilbert (cd.) (New York, NY: Reinhold, 1961), pp. $282-2 \%$.

8K.E. Drexler, "Molecular Engineer@: An Approach to the Development of General Capabilities for Molecular Manipulation," Proceedings of the National Academy of Science USA, vol. 78, No. 9, September 1981, pp. 5275-5278.

${ }^{9}$ Rolf Landauer, "Poor Signal to Noise Ratio in Science," Dynami “c Patterns in Complex Systems, J.A.S. Kelso, A.J. Mandell, M.F. Shlesinger (eds.) (Singapore: World Scientific, 1988), pp. 388-394.

${ }^{10}$ Eric Drexler, Foresight Institute, personal communication, March 1991.

${ }^{11}$ In the United States, one researcher is Performing molecular modeling at Xerox Palo Alto Research Center. A nonprofit organization, Institute for Molecular Manufacturing, in Palo Alto, California was formed this year and plans to sponsor research in the future. No projects in Japan or Europe are explicitly directed at molecular machine development, although the Hotani Molecular Dynamics project sponsored under the Japanese Exploratory Research for Advanced Technology (ERATO) program addressed molecular machine concepts in addition to its regular line of scientific investigation. 
Figure 24-Three Approaches to Electronics Miniaturization

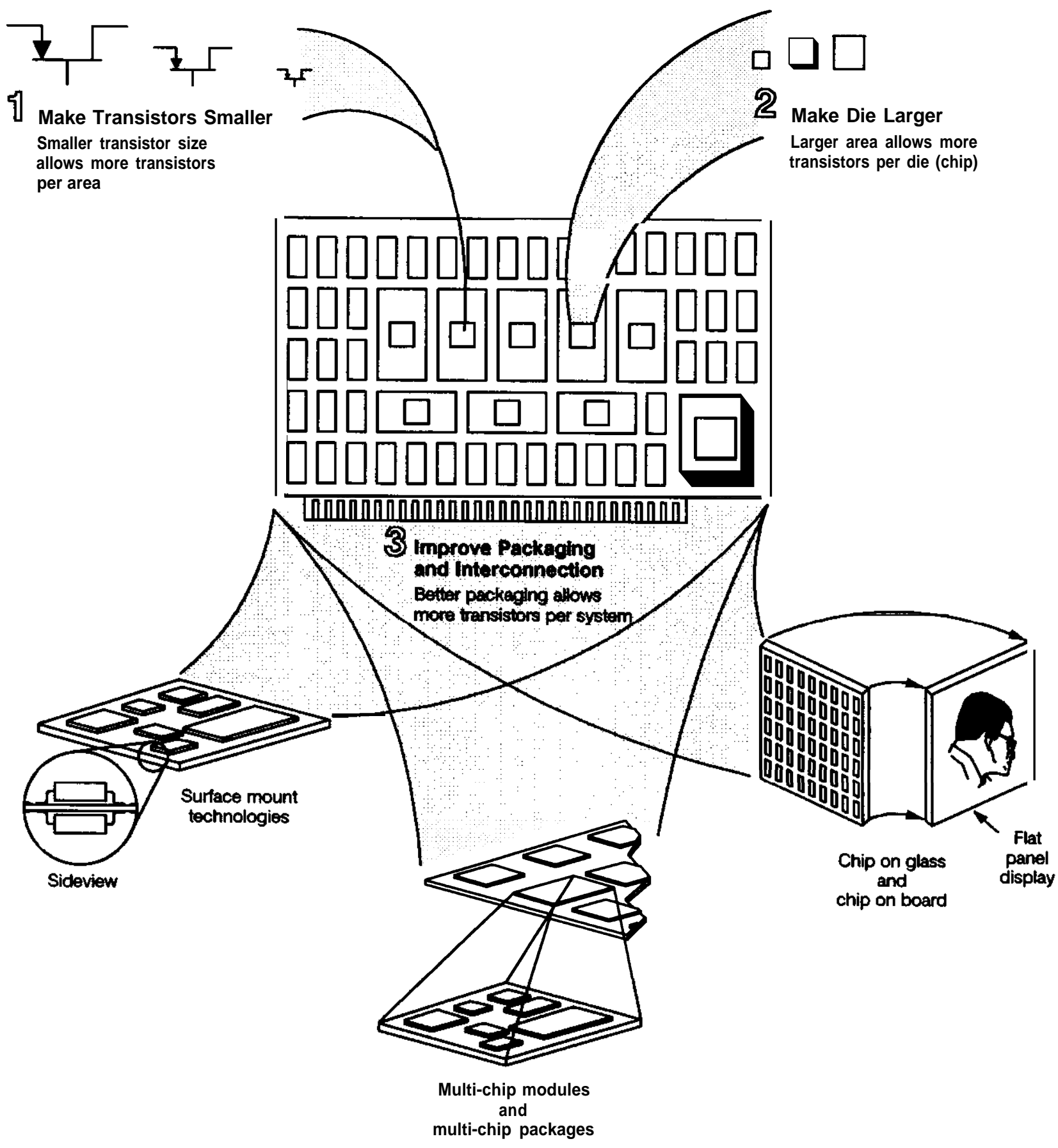

Three basic strategies areused to miniaturize electronics systems. By making each transistor smaller (I), more can be placed on an integrated circuit, incorporating the functions of other chips. Increasing the size of the chip (2) has a similar effect by increasing the available area for transistors and their interconnections. These first two approaches have been the driving force behind electronics miniaturization for at least the past two decades. Improved packaging (3) is a way to improve use of space on a printed circuit board. Atypical printed circuit board has only a small fraction of its space covered with integrated circuits; the rest is primarily packaging and interconnection.

SOURCE: Office of Technology Assessment, 1991. 


\section{Display Technology}

The size of a typical cathode ray tube (CRT) desktop computer display is about a cubic foot. New technologies, e.g., as liquid crystal display (LCD) and electroluminescence displays, have allowed the development of flat panel displays that occupy a tenth of the volume. Flat panel display technologies are discussed in detail in the OTA report The Big Picture: HDTV \& High Resolution Systems. New technologies required to advance high-resolution video technology are many of the same that are required for advanced electronics and more powerful computer systems. ${ }^{8}$ Japanese producers of flat panel displays favor LCD technology and are increasingly adapting it to larger screens, but current fabrication techniques are limiting screen size to about 15 inches. Further increases in screen size await improved lithography.

\section{Data Storage}

"Mass Storage" refers to storage technologies with large capacities, including optical and magnetic media that retain data when the power is off. These devices encode data on a surface that can change its magnetic or optical characteristics. By moving the surface under a device that can sense and change the characteristics of the surface-a $\mathrm{read} / \mathrm{write}$ head-data is stored and retrieved.

Semiconductor memory storage increases capacity as constraints on transistor miniaturization can be overcome, but mass storage operates on different physical principles and therefore has different limitations. There are two major types of mass storage technologies-magnetic and optical. The trends in magnetic storage show no sign of slowing as they approach densities that will put
1 billion bits onto 1 square inch. Researchers at IBM's Almaden Research Center have demonstrated such densities. ${ }^{9}$ Today's storage densities are about 50 to 100 million bits per square inch in high-end storage systems. Optical storage technology uses lasers to write and read data from an optical disk and is limited by the size of the laser beam spot on the disk. The spot size, in turn, is limited by the wavelength of the laser source. Most optical disks today use a red (wavelength of 800 nanometers) laser to write and read data. Efforts are underway now to develop blue lasers (wavelength of 400 to 500 nanometers) that would have smaller spot sizes and be able to increase the capacity of optical disks by four times.

Another technique that promises much higher densities uses STM $^{10}$ to write and read data. The STM and related instruments use sharp tips in close proximity to a surface to create an atom-byatom image of the surface. These tools also have the capability to physically modify surfaces by either etching away or building up a few hundred atoms at a time. Such techniques could result in massive data density-more than 1,000 billion bits $(1$ terabit $)$ per square centimeter. The major problem with STM and related approaches to data storage is that the data access speed is painfully slow." There is research currently underway at a few labs to combine many tips in parallel, increasing the speed of writing and reading. These approaches remain speculative, but might yield useful technology in the long term.

\section{Interconnection and Packaging}

The basic packaging component of most computer systems is one or more circuit boards. Various components are placed on the circuit board and interconnected with strips of metal. Due to manufacturing costs, the number of components

\footnotetext{
${ }^{8}$ The techniques used to make LCD displays are many of the same that are used to make integrated Circuits. For more information see OTA's Background Paper, The Big Picture: HDTV \& High-Resolution Systems, OTA-BP-CIT-64 (Washington, DC: U.S. Government Printing Office, June 1990).

${ }^{9}$ Robert M. White, "Peripherals," IEEE Spectrum, February 1990, pp. 28-30.

${ }^{10}$ See app. A for details on the STM.

non $_{\text {e estimate is that }}{ }^{32}$ centuries would $\mathbf{b}_{\text {e }}$ required to write one square centimeter with one proximal probe. See James S. Murday and Richard J. Colton, "Proximal Probes: Techniques for Measuring at the Nanometer Scale," in Chemistry and Physics of Solid Surfaces, R. Vanelow and R. Howe (eds.) (New York, NY: Spnnger-Verlang, 1990), p. 347.
} 
is the greatest contributor to the overall cost of an electronics system (see figure 2-5). System designers seek to reduce the number of components on a board to improve reliability as well. The interconnecting strips of metal on a board and connections between boards are more likely to break than connections elsewhere in the system. Ever since the invention of the integrated circuit, the best way to reduce costs and increase reliability is by putting more transistors on integrated circuits, hence reducing the number of circuit board components. By decreasing the size of each transistor and their interconnections, more transistors have been crammed onto integrated circuits.

While the density of circuits on a chip have been increasing, the size of the chip has been increasing as well. The first microprocessor in 1971 was on a 19,000 square $\mathrm{mil}(1 \mathrm{mil}=$ onethousandth of an inch) chip. For the next 20 years, chip size increased by about 14 percent per year. Current microprocessors are on chips of about 260,000 square roils. Chip size may rise even faster over the next few years, yielding a chip size of 625,000 square roils in production by $1994 .^{12}$ Since the probability that a chip is defective increases with its size, chip size is limited by the number of defects on a wafer (see figure 2-6).

If the increase in chip size is taken to its limit, an entire wafer can become one large circuit. Since a typical wafer produces about a hundred chips, a huge number of transistors could be put on one wafer. But there are significant problems with such an approach. There are defects in every wafer (primarily caused by particulate from the manufacturing equipment), so a wafer-scale circuit is virtually guaranteed to have defects. With the high defect rate, strategies for routing connections around defective components must be considered in circuit design. Another problem is that the complexity of interconnecting wafer-scale circuits increases the number of processing steps.
Finally, the fine lines of metal used to link the imbedded transistors together are not suited for the relatively long interconnections needed for wafer-scale integrated systems. There are some specialized applications that could use waferscale integration such as image processing and large memory storage, ${ }^{13}$ but unless fabrication problems are solved, it will likely not be an important technology. Applications that have regular designs - e.g., memory is essentially the same circuit pattern repeated over and over-have achieved marginal success with wafer-scale integration because errors in the circuit are relatively easy to detect and correct or bypass.

Hybrid wafer-scale integration-known as multichip module (MCM) - is another approach to increasing circuit density. MCMs take several chips, place them on a substrate (usually silicon or a ceramic), and connect them with thin films of conducting metal using techniques like those used in integrated circuits. Multichip modules place chips closer together than is possible using single chip packages - an important advantage as chip speeds increase. The distance a signal can travel in one clock cycle-the "heartbeat" of a computer system-shrinks as cycle times shorten. Machines with clock cycles of 2 nanoseconds will soon be available, ${ }^{14}$ which corresponds to 500 megahertz. (The fastest of today's personal computers operate at about 33 to 50 megahertz.) Reliability is also enhanced because the number of printed circuit board connections and board-toboard connections are reduced.

MCMs have been successfully used in the IBM 3090 and the NEC SX-AP; high manufacturing costs, however, keep them from being used in mass production items like personal computers and workstations. ${ }^{15}$ The Microelectronics \& Computer Technology Corp. (MCC)--a private consortium of electronics companies-has developed a MCM technology capable of producing

\footnotetext{
12Jerry Sullivan, "The Next Generation of Electronics Design Automation Technology for Systems in Silicon," MCC Technical Report No. CAD-043-90, Jan. 24, 1990.

13"A Dream Remembered," The Economist, Nov. 17, 1990, p. 12.

${ }^{14}$ Dennis Herrell and Hassan Hashemi, "Hybrid Wafer Scale Integration," MCC Technical Report P/I-329-89, 1989.

${ }^{15}$ Ibid.
} 
Figure 2-5-Relative Cost of a Chip at Different Levels of Interconnection

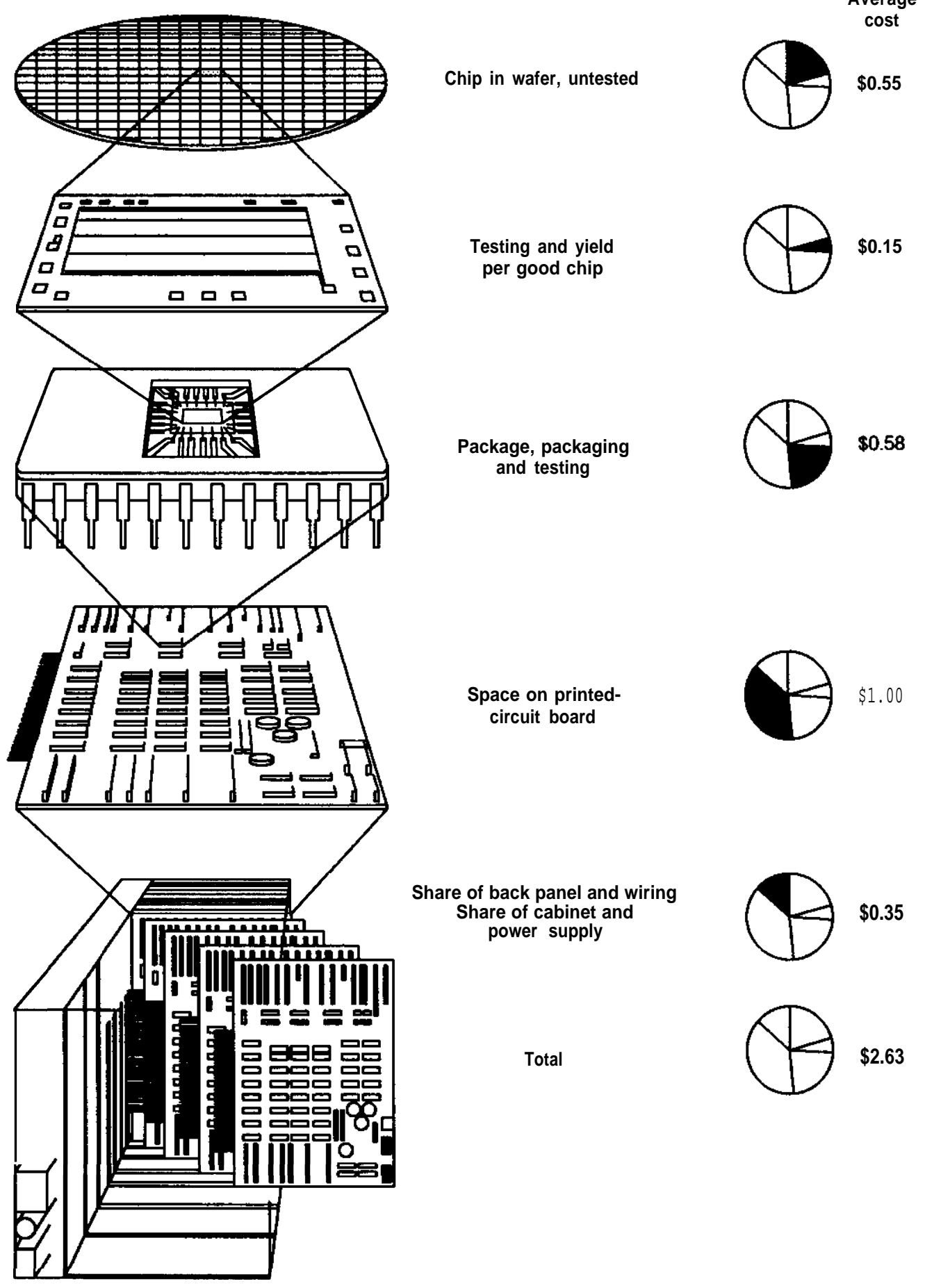

Space on the printed circuit board is one of the most expensive commodities in a system design. By integrating more functions onto a chip, system designers can reduce the number of chips on board, lowering overall system cost. The illustration shows the steps that a chip goes through on its way to becoming part of a computer system and the relative cost per chip for each stage.

SOURCE: Adapted from Ivan E. Sutherland and Carver A. Mead, "Microelectronics and Computer Science," Microelectronics (San Francisco, CA: W.H. Freeman \&Co., 1977), p. 113. Copyright(c) 1977 by Scientific American, Inc. All rights reserved. Data from Graydon Larrabee. Texas Instruments, personal communications, Sept. 20, 1991. 
Figure 2-6- Relationship of Die Size and Yield
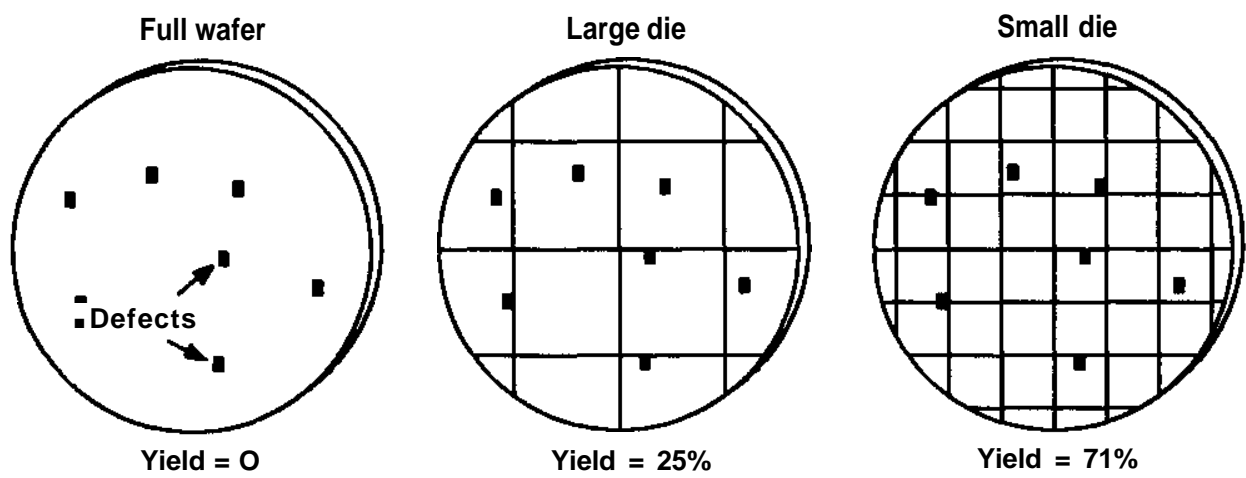

Smaller die (chip) size means higher yield (percentage of good chips) for a given density of defects. SOURCE: Adapted from Sand, Silicon, Systems, Howard K. Dicken (cd.) (Scottsdale, AZ: DM Data Inc., 1986), p. 7-12.

computers that are fast ( 3 nanosecond cycle time, or 333 megahertz), use 10 million gates, and cost $\$ 5,000$. Costs must be reduced further for the technology to be affordable for workstations and personal computers.

A major obstacle to widespread use of MCMs is the lack of standardized interfaces for the hardware and software needed to design the modules. For the last 30 years the electronics industry has been building an infrastructure that is focused on the same objective: improving the performance of the integrated circuit. All the manufacturing equipment and computer software is geared for designing and optimizing a single chip. It is difficult to obtain "naked" chips (dice) outside their package from semiconductor vendors. Computer-aided design and testing are all difficult with MCMs.

Dissipating the heat generated by dense circuitry is a major problem facing circuit designers. This becomes more difficult as the number of transistors in a chip increases. The simplest approach to accomplish this in a computer system is to flow air over the circuits. In high-performance systems (supercomputers and some mainframes), however, where speed is critical, other means for cooling are often used. Some supercomputers flow inert fluids, e.g., freon, directly over the circuitry. Sometimes fluid distribution systems attached directly to the chip package are used to dissipate heat, a scheme used in several mainframe computers.

Many options for increasing the density of electronic circuits now being investigated operate best at very low temperatures and require external refrigeration units. Advancements in refrigeration and heat dissipation technology are important to pushing the miniaturization of electronics devices. This is because as the cross-section of a conductor decreases, the resistance to current flow rises. Greater resistance means greater heat generation because the same current flowing through a high resistance material creates more heat than in a low resistance material.

The need for better interconnection is greatest in two areas: 1) consumer electronics and 2) highperformance computers. Consumer electronics must combine low costs with high dependability. High-performance computers demand ultra-fast computing speeds and extensive interconnection; while the field is highly competitive, cost is less a factor than performance. In high-performance computing, the need for better interconnection results from the short distance that an electronic signal can travel before another clock cycle starts. Supercomputers push clock cycles to the limit, so designers must devise ways to minimize interconnection distance. The Cray supercomputers have an unconventional circular shape in order to keep components close together and reduce the time 
needed to communicate among components. Massively parallel computers face an even more serious interconnection problem because many more components (processors) must be connected. A conventional serial (von Neumann architecture) mainframe has only one central processing unit (CPU) that is centrally located so that other components can be closely connected to the CPU. A massively parallel computer may have over 1,000 CPUs that must be interconnected.

In consumer electronics, a major objective is to make the product smaller, lighter, cheaper, and more reliable. Each of these objectives demand better interconnection and packaging. Japanese industry, with its emphasis on consumer electronics, tends to adopt packaging innovations to meet the demands of the market. The impact can be seen in the many consumer products around us (see figure 2-7).

\section{SENSORS}

Sensors are devices that can monitor and translate observed conditions-light level, acceleration, pressure, or temperature-into a signal. The signal can then be transmitted, processed through a system or stored as data. A mercury thermometer, for example, responds to an environmental condition-temperature-and translates to a visual readout indicated by the level of mercury gauged against a calibrated scale. An electrical temperature sensor might sense the change in temperature by measuring the change in voltage across a material. The resulting electrical signal can be manipulated and displayed for read-out.

There are a wide variety of sensors; each can be classified as imaging or non-imaging. ${ }^{16}$ Imaging sensors take many measurements of radiated energy from the imaged target. Camcorders, for example, use a charge coupled device (CCD) as an imaging sensor to convert a visible light image to an electrical signal that can be recorded on a
Figure 2-7-impact of Surface Mount Technologies on Calculator Thickness

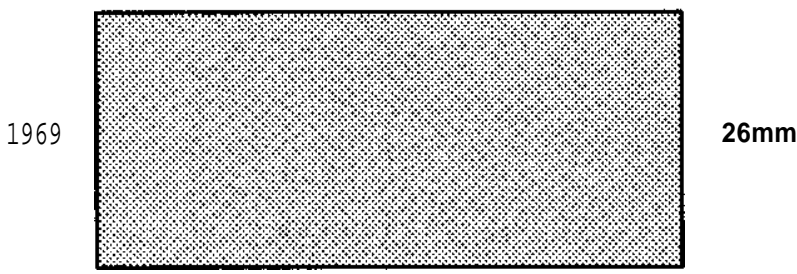

1973



$13 \mathrm{~mm}$

1975

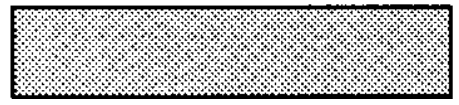

$9 \mathrm{~mm}$

1976

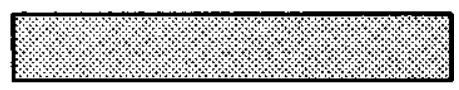

$7 \mathrm{~mm}$

1977

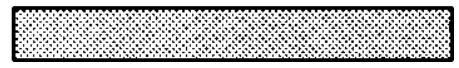

$5 \mathrm{~mm}$

1978

$3.8 \mathrm{~mm}$

1979

$1.6 \mathrm{~mm}$

Implementing surface mount technologies in calculators had a dramatic impact on thickness. Surface mount technologies include surface mounted packages as well as surface mounted keyboards and displays.

SOURCE: VLSI Research.

VCR tape. Non-imaging sensors act at a single point, typically in contact with the object being sensed. Sensors can be made to sense different substances and energies: electromagnetic radiation, temperature, pressure, acceleration, chemicals, and biological materials. Of the many sensor technologies, a few are key to miniaturizing systems: CCDs, chemical and biological sensors, and micromechanical sensors.

\section{Charge Coupled Devices}

CCDs are solid-state image sensors that detect light. They are used in cameras-from camcorders to professional cameras to astronomical cam-

\footnotetext{
${ }^{16}$ Exceptions t. this classification include some tactile sensors that detect sensations of touch with an array of discrete sensors.
} 
eras on satellites. The resolution of a CCD is determined by the number of picture elements (pixels) on a CCD. The devices are a special kind of integrated circuit with problems similar to other integrated circuit manufacturing. Reducing the size of each pixel improves the pixel density and the image resolution. Increasing the size of the CCD can increase the number of pixels, but defects in the circuit cause yield to plummet as the CCD size increases. Manufacture of CCDs is dominated by Japanese industry. More than 20 Japanese companies manufacture CCD chips and they are the leaders in small pixel size, and innovative design. ${ }^{17}$ In the United States, Tektronix, Texas Instruments, and Kodak produce CCDs designed for military, industrial and space applications. The majority of the image-sensing market is in consumer electronics. ${ }^{18}$

Imaging sensors are particularly important to the Department of Defense (DOD). DOD relies on imaging data for things like aiming missiles, detecting rocket launches, and enhancing nightvision. Most DOD applications sense areas of the electromagnetic spectrum that are unique to military needs.

\section{Chemical and Biological Sensors}

By combining information technologies with biotechnologies, researchers are developing new sensors that are cheaper, faster, more versatile, and more efficient than previous generations. These sensors, known as biosensors, can detect gases, chemicals, and biological molecules. The first biosensor was invented in $1 \% 2$ by Leland $\mathrm{C}$. Clark Jr., but it was not until the 1970s that biosensors came into practical use. The early biological and chemical sensors were relatively large; Clark's first glucose sensor was about a centime- ter in diameter. ${ }^{19}$ There are many ways to make biological and chemical sensors; some can be made using techniques borrowed from integrated circuit manufacturing. By applying this technology, it is now possible to make sensors that are only a few thousandths of a centimeter wide.

Smaller biosensors have two advantages: 1) they can be placed in areas that were previously inaccessible, and 2) they are fast since the measurement can be done in the field instead of at a central lab. Because they offer similar economies of production as microelectronics, biosensors will become cheap and widely available. Biosensors will be useful in portable systems. A market analysis by Arthur D. Little, Inc. in 1991 predicted that portable biosensors sales would reach $\$ 1$ billion by $2000 .{ }^{20}$ With sensors for substances like glucose, urea, and carbon monoxide available in an inexpensive and small package, portable diagnostic kits could be made available (see box $2-\mathrm{B})$. The industrial food processing industry is expected to make use of portable biological sensors to determine the freshness of food. Because biosensors are small enough to be placed on the tip of a hypodermic needle, blood chemistry could be monitored continuously by placing several biosensors onto a chip inserted on a catheter tip into the patient during surgery. The capability to measure chemicals concentrations will be useful for process industries such as biotechnology and chemical production.

Miniaturization of biosensors and chemical sensors relies on a device called a chemical field effect transistor (ChemFET). ${ }^{21}$ ChemFETs are similar to the field effect transistors (FET) that are used in normal microelectronics. A microelectronics FET conducts electricity (turns on) when a voltage is applied to one of it inputs (gate), creating an electric field in the FET. A ChemFET

\footnotetext{
${ }^{17}$ U.S. Department of Commerce, "JTECH (Japanese Technology Evaluation Program) Panel Report on Advanced Sensors in Japan," January 1989 , p. 40.

${ }^{18}$ Ibid., p. 44.

${ }^{19}$ Jerome S. Schultz, "Biosensors," Scientific American, August 1991, pp. 64-69.

20"Strong Growth for Biosensors," New Technology Week, Feb. 19, 1991, p. 8.

${ }^{21}$ Also known as ion sensitive field effect transistor (ISFET). "CHEMFET" isusuallyused to refer to sensors that use whole molecules, while "ISFET" refers to sensors designed with ions (charged atoms or molecules).
} 


\section{Box 2-B--Bedside Analysis}

A hand-held analyzer currently under evaluation at the Hospital of the University of Pennsylvania demonstrates how biosensors might find their way into clinical use. It simultaneously makes six commonly requested chemical measurements on a patient's blood-sodium, potassium, chloride, urea nitrogen, glucose, and hematocrit-producing results in less than 2 minutes. The bedside tests cost more than ones performed in a central laboratory, but their immediacy may make them more effective.

The device achieves accuracy comparable to that of laboratory equipment by using a disposable cartridge containing six biosensors and a calibration sample. A medical worker places 60 microliters of blood in the cartridge; the analyzer then measures both the calibration sample and the patient sample. It displays test results and also stores them, keyed to time and the patient's identification number, for later analysis: The cartridgebased design adopted by manufacturers will make it possible to perform a different set of tests once the appropriate sensors have been developed.

'Adapted fro ${ }_{\mathrm{m}}$ Jerome Schultz, "Biosensors, Scientific American, August 1991, pp. 64-69.
Figure 2-B-1 -Bedside Analysis
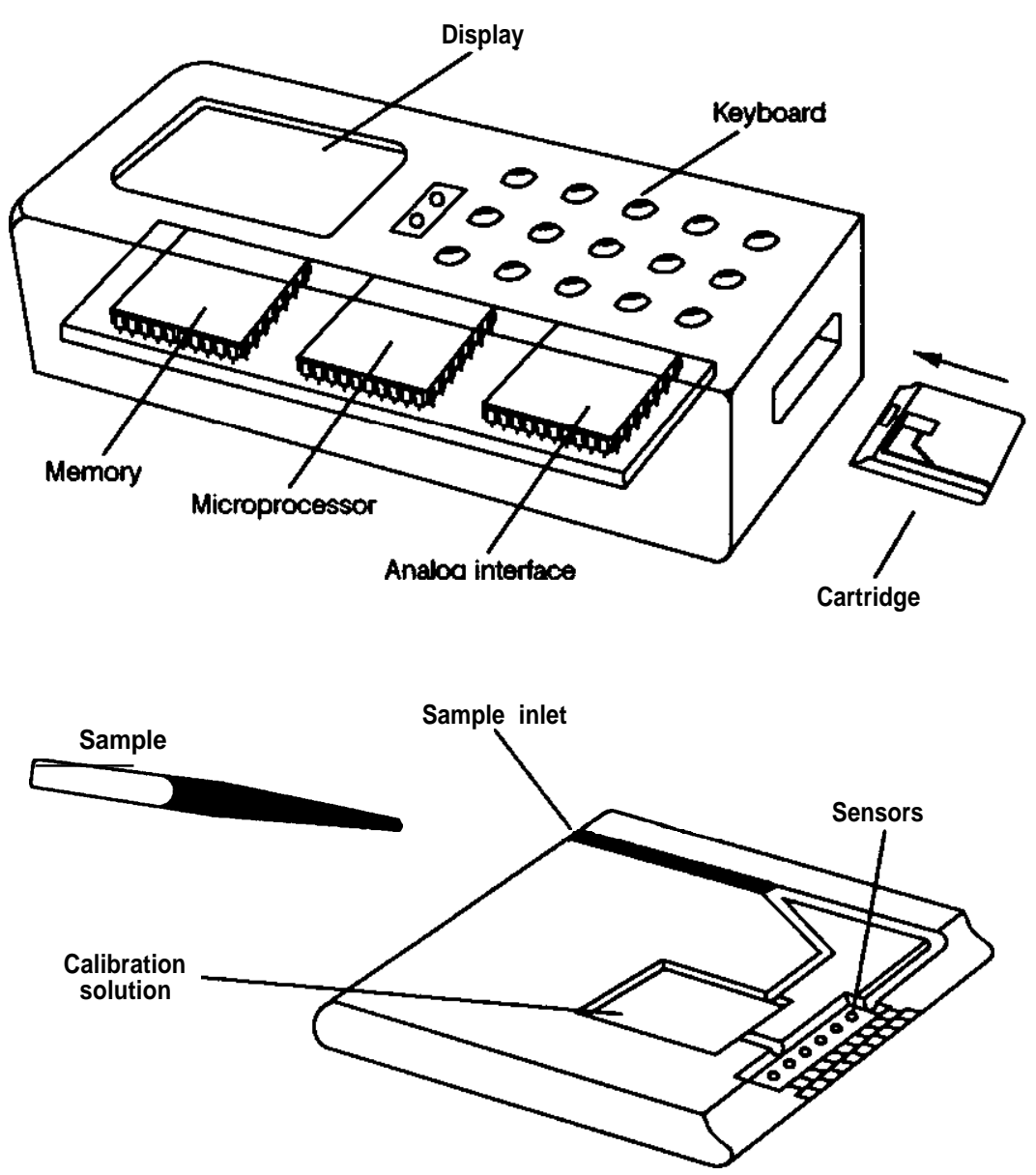

SOURCE: Adapted from Jerome S. Schultz, "Biosensors." Scientific American, August 1991, pp. 64-69. Copyright (c) 1977 Scientific American, Inc. All rights reserved. uses a FET that is specially coated with a chemical or molecules that will create an electric field when the sensor is exposed to a specific chemical, gas, or molecule.

Fiber-optics technology aids biosensor and chemical sensor development and miniaturization. Certain chemical and biological molecules will change their optical properties (either give off or absorb light differently) when exposed to other chemicals or molecules. For example, the enzymes dehydrogenase and luciferase react with testosterone, the male hormone, to produce a secondary chemical that gives off light (fluoresces). A fiber-optic-based glucose sensor might one day serve as the basis of an implantable artificial pancreas. In the meantime, glucose sensors are being incorporated into the latest generation blood sugar monitors for diabetics. These devices are portable, take measurements in seconds compared to hours with earlier monitors, and some function without drawing blood by measuring absorption of light through skin.

\footnotetext{
${ }^{22}$ Schultz, op. cit., footnote

23J. Travis, "Helping Diabetics Shed Pins and Needles," Science News, vol.140, No. 1, July 6, 1991, p. 4.
} 
New generations of biosensors and chemical sensors will take advantage of theircommon heritage within formation technology to integrate logic functions into the same package with biosensors. Integrating logic with sensors can result in more useful devices. For example, a glucose sensor with integrated logic might respond differently to blood sugar levels at different times of day. Integrated circuit fabrication holds promise for high integration by placing biosensors and electronics on the same chip. It also promises reduction in prices because large numbers of sensors can be fabricated at one time. Major challenges remain, however, such as isolating the microelectronics from the environment while allowing the sensor access to the environment.

Researchers are actively pursuing refinements to the fabrication processes that will make widespread use of biosensors possible. The yield and uniformity of the manufacturing process needs to be improved before large scale production is practical. Shelf life is a problem with biosensors; compared to microelectronics and fiber optics, biosensors are perishable. Biomolecules that must be affixed to a chip or optical fiber are not stable outside their cellular environments. As a result, biosensors can only be stored for weeks or months-sometimes years. This limitation prohibits some applications, but for some industries short lifetime products are normal and expected. In medicine, for example, instruments are often discarded after use to avoid risk of contamination. The price and capabilities of biosensors in other applications (e.g., detecting explosive gases or critical chemicals in a process) are valuable enough that users are willing to accommodate limited lifetimes.

\section{Micromachined Silicon Sensors}

A significant field for future sensor developments will be mechanical sensors-devices that rely on the mechanical properties of a material to sense energy of their surroundings. Since 1958, silicon has been used in pressure sensors. By fabricating a covered cavity, changes in ambient conditions can be detected by monitoring the resistance across resistors on a membrane covering the cavity (figure 2-8). Improvements in silicon processing technology have reduced the size and cost of silicon pressure sensors, with major gains made during the 1980s. Cost has gone from about $\$ 1,000$ per sensor in the 1960 s to a few dollars per sensor today. The 1958 silicon pressure sensor that was half an inch wide is now one hundredth of an inch wide. Growth in the 1980s was very strong; silicon pressure sensors are 60 percent of the pressure sensor market - up from 40 percent in 1985 and 16 percent in $1980 .^{24}$

Silicon mechanical sensors are also used to detect acceleration. Accelerometers are made with a mechanical silicon structure that places a mass of material-the "proof mass" —on the end of a thin "arm" of silicon. Acceleration makes the

Figure 2-8-Cut-away View of a Silicon Pressure Sensor

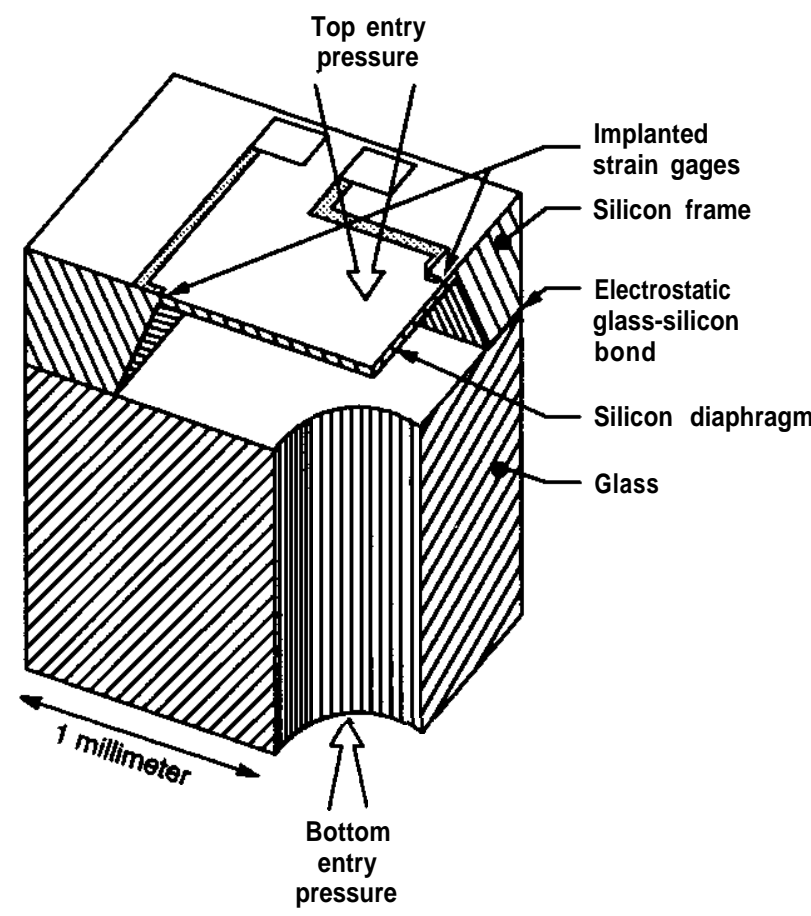

SOURCE: Adapted from Janusz Brysek et al., Silicon Sensors and Microstructure (Fremont, CA: NovaSensor), 1990, p. 7.3 .

\footnotetext{
24Janusz Brysek et al., Silicon Sensors and Microstructures (Fremont, CA: NovaSensor, June 1990), p. 2.18.
} 
silicon arm bend; the degree of bending determines the acceleration. Accelerometers are finding applications in automobiles for airbag deployment and automatic suspension systems. Analog Devices, Inc., for example, is using a surface-micromachined cantilever as the basis for a accelerometer. The separation between the cantilever and the chip surface changes with acceleration, changing the voltage. The voltage change can be translated into an acceleration measurement.

The miniaturization of silicon sensors resulted from developments in micromachining-a technique to precisely shape the surface of a material such as silicon. Silicon in its crystalline form has different orientations in the crystal that can be specified for a particular silicon wafer. Chemicals used to etch silicon "eat away" crystalline silicon at different rates depending on orientation of the crystal to the surface. This process is called preferential etching. By combining preferential etching with special chemicals that stop short the etching process, complex structures can be created in silicon. This technique is known as "bulk micromachining," and has been used since the 1960s.

Use of "sacrificial layers"-technique developed during the 1980s--can create more intricate structures. Sacrificial layers are thin films (usually less than 10 microns) that are removed by etching chemicals to release movable parts from the substrate (see box 2-C). The process of using multiple layers of sacrificial layers to create complex structures is called surface micromachining. Using surface micromachining, researchers at the University of California at Berkeley and at AT\&T Bell Labs fabricated a working motor about the width of a hair (100 microns) in 1988 (see photograph). That achievement set off a flurry of research activities. Laboratories around the world were soon duplicating and improving upon the original work. Work at the Massachusetts Institute of Technology (MIT) improved the sacrificial layer technique, making it more compatible with traditional silicon electronics processing. The Karlsruhe Nuclear Research Center in Germany developed a variation on the surface- micromachining technique known as "LIGA." It uses $\mathrm{x}$-ray lithography technologies to fabricate microcomponents from other materials including plastic and metal.

The new complex structures have created a need for better understanding and characterization of the materials used in the structures. Silicon is a well known material, but not enough is understood of its mechanical properties. Structures that are released from the substrate, for example, can warp due to strains in the material. In microelectronics these strains are not important because structures remain connected to the substrate, but for surface micromachining, these strains can ruin a component. Wear and friction are not well understood at such small scales, and researchers need to understand these properties to improve the reliability of micromotors and other movable parts. Characteristics of surface interactions, flow of fluids, and air flow are not well understood at such small scales. Under-

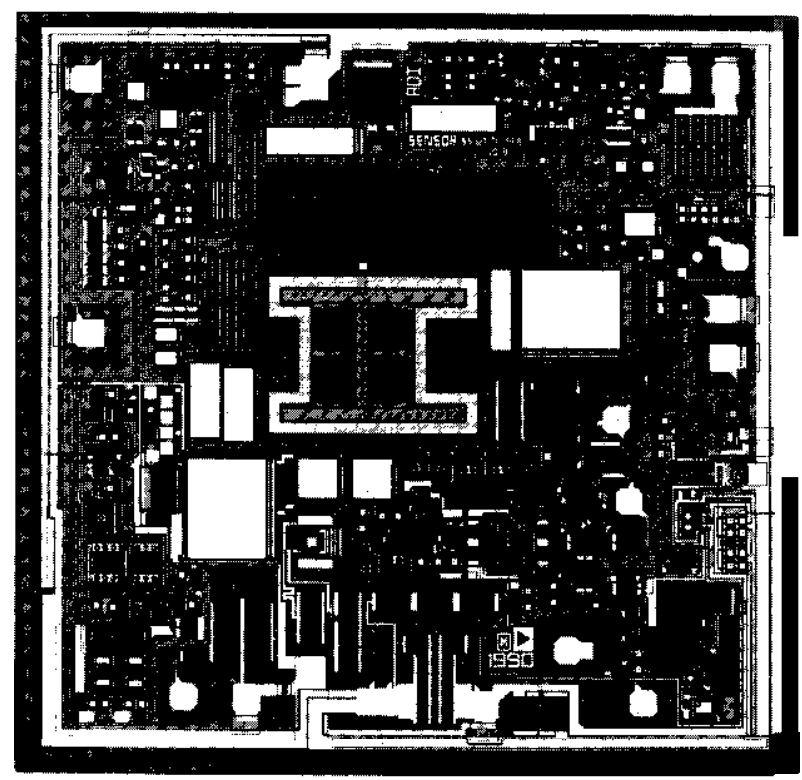

Photo credit: Analog Devices, Inc.

An acceleration sensor (accelerometer) made by Analog Devices, Inc. will be the first commercial product that uses surface micromachining, a new processing technique that uses sacrificial material layers to free structures from a substrate. The microstructure, similar to ones fabricated at the Berkeley Sensor and Actuator Center, can be seen in the center of this microphotograph of the sensor chip. The sensor is surrounded by electronics that provide calibration and signal conditioning. 


\section{Box 2-C--A New Way To Machine}

1. The pattern of the gear is transferred to the substrate by shining ultra-violet or $\mathrm{x}$-ray light through a stencil-like mask.

2. This sequence is repeated several times to achieve a structure that has alternating layers of silicon and "sacrificial layers." (See the description of fabrication technologies in this chapter and app. A for details.)

3. The sacrificial layers can be dissolved in a chemical that doesn't disturb the silicon. After the sacrificial layer is removed, the gear is free to rotate. A restraining hub prevents it from flying off the surface of the chip.

\section{Figure 2-C-1 -Surface Micromachining}

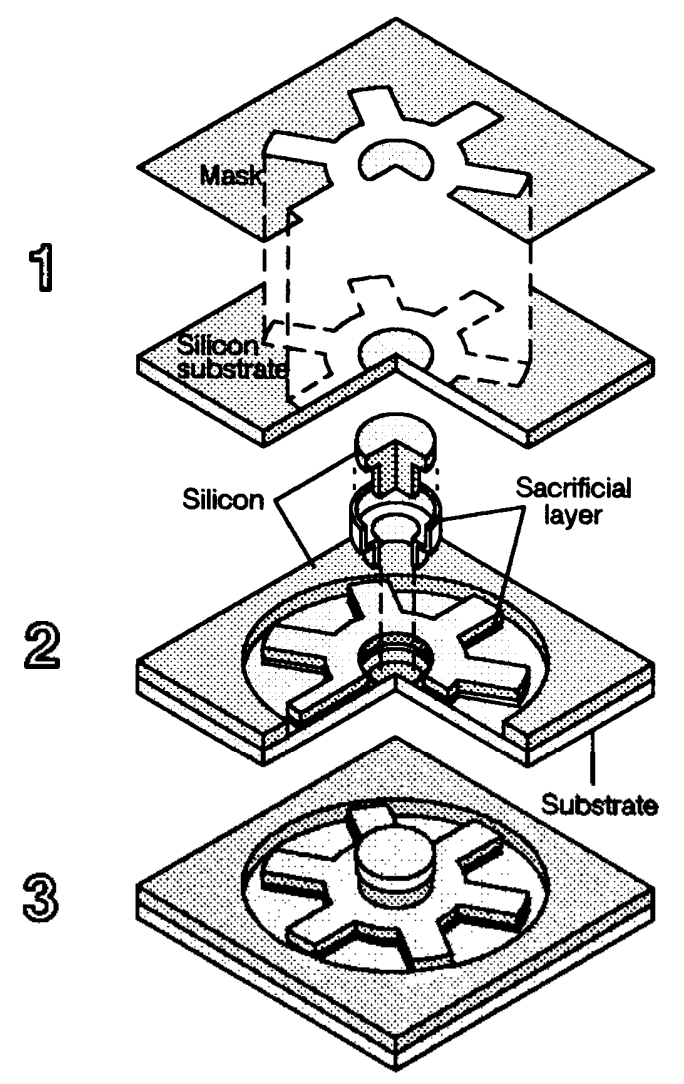

SOURCE: Sharon Begley "Welcome to Lilliput," Newsweek, vol. 11.7. No. 5, Apr. 15, 1991, pp. 60-61. Artist Jared Schneidman. Copyright (c) 1991, Newsweek, Inc. All rights reserved. standing fluid flow at such small scales will be important if the technology is used for chemical and biological processing applications.

Future progress in micromachined sensors are in three areas of improvement:

1. design and fabrication processes will improve performance and expand applicability;

2. manufacturing technology will become more important as sensors become commodities; and

3. packaging of the sensor is the third challenge, since reduction in packaging costs has a significant impact on cost of the final product.

Manufacturing technologies and procedures have received insufficient attention in the past. ${ }^{25}$ This could hold particular peril for U.S. industry if it is not remedied as it competes with Japanese companies attuned to the value of robust manufacturing methods.

\section{ACTUATORS}

Actuators translate a signal to motion or force. A solenoid valve is a common type of actuator that relies on electromagnetic forces to move a plunger arm to open and close a valve. Electrostatic actuators are promising small-scale actuators because electrostatic fields can easily be created in micro-structures and are relatively powerful at small scales. Recent developments indicate electromagnetic actuators are promising as well. Small actuators have also been made from piezo-electric material ${ }^{26}$ and shape memory alloys. $^{27}$

\section{Micro-Electro-Mechanical Actuators}

Surface micromachining techniques are used to fabricate micro-structures with moving parts.

\footnotetext{
${ }^{25}$ Ibid., p. 2.18.

${ }^{26}$ Piezo-electric materials contract or expand when a voltage is applied.

${ }^{27}$ Shape memory alloy materials change shape with a change in temperature.
} 
Researchers at the University of California at Berkeley and AT\&T Bell Labs fabricated motors in 1988 using these new techniques. These motors are typically about 100 microns in diameter (the width of a hair) and about 3 microns deep. Minuscule motors have also been fabricated at University of Utah and AT\&T Bell Labs using more traditional machining techniques that are as small as 500 microns in diameter. These motors produce more power than the smaller motors produced by surface micromachining and might be useful in applications such as microsurgery or drug delivery. ${ }^{29}$ Small actuators could also be used in consumer items, (e.g., cameras, cassette players, video recorders, and toys) robotics and defense applications.

Tiny levers, gears, and other mechanical assemblies can be etched from silicon with the same surface micromachining techniques used to make motors. Gears, levers, and the like might be useful for transmitting motion and force. At such small scales, these mechanisms might be useful formanipulating light, low-mass objects. There are many potential applications in optics; several materials that can transmit, emit, or reflect light can also be used in the construction of microactuators. Tiny mirrors on movable levers or gears could serve as optical switches or light modulators by moving from one position to another. Moving chemicals, fluids, and cells is currently the object of much research. Electric fields and ultrasonic waves are demonstrated techniques that are being refined through research and development at university and industrial laboratories. Hewlett-Packard and Canon use bulk-micromachined devices to dispense ink for their thermal ink jet printers. ${ }^{30}$

\section{Integrating Actuator, Sensors, and Electronics}

One of the most exciting aspects of making tiny structures in silicon and other materials is the prospect of combining sensors, actuators, and electronics into integrated systems on a single chip. The greatest prospect for near term applications is probably science and engineering instrumentation.

Chemical and biological applications also appear promising: small tweezers might be useful to hold specimens (e.g., cells) in place while they are manipulated by other devices, such as injection needles. Drug delivery systems might be made small enough to be worn by patients, or even inserted into the body. Chemical processing that often uses complex lab equipment might be performed on a chip or wafer in a portable system. Biotechnology procedures normally performed in a batch process could be done on a cell-by-cell basis with greater control over the results.

At Lawrence Livermore Labs in California, researchers are working on spectrometers that could be used to monitor environmental hazardous materials. Lawrence Livermore is also developing a DNA sequencer on a chip. At least one major U.S. company is developing a gas chromatography system on a chip. Hitachi announced in 1989 that it had a prototype cell fusion system ${ }^{31}$ that fused as many as 60 percent of cells compared with about 2 percent using conventional techniques.

Looking even further into the future, "microrobots" might be fashioned from micromechanical components. These tiny robots could perform a variety of functions. One of the objectives of the MITI micromechanics program is to develop microrobots that would be capable of inspecting inaccessible or hazardous locations such as in jet engines, nuclear plants, and the human body. Here in the United States, similar research is being conducted at NASA's Jet Propulsion Laboratory and a few universities. The objective of the NASA program is to make microrobots and microrovers useful for exploration of other planets and the moon.

\footnotetext{
28"Techniques include extrusion, diamond-point machining, and EDM (electro-discharge machining).

${ }^{29}$ Small motors of this type are already being used for drug delivery for livestock.

${ }^{30}$ Phillip Barth, Hewlett Packard, personal communication, May 29,1991.

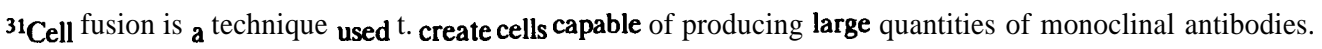




\section{INTRODUCTION}

Many of the technologies that have enabled advances in miniaturization were first developed for microelectronics and allow both lateral and thickness control in the creation of structures. Although the early techniques and tools were directed at silicon, recent years have seen increased attention to materials other than silicon: compound semiconductors, superconductors, metals, and insulators. Furthermore, they are being applied to more diverse areas: micromechanical structures, biosensors, and chemical sensors.

The same processes that have allowed for decreases in size also allow parallel production of many devices. Thousands or millions of transistors or other devices can refashioned simultaneously on one chip the size of a thumbnail, and many chips preprocessed at the same time on one wafer. The key steps in the process of creating these structures are:

- lithography,

- pattern transfer, and

- characterization.

\section{LITHOGRAPHY}

Integrated circuit fabrication techniques all rely on lithography, an ancient technique first used for artistic endeavors. The basic process involves covering an object with a thin layer of material (ancient artisans sometimes used wax) that can be patterned but will resist subsequent processing and protect the material underneath. Industrial lithographers use a hydrocarbon polymer, appropriately called "resist." A pattern is produced in the resist-usually by exposure to visible light or ultraviolet (UV) radiation - exposing selected areas of the material below. The exposed areas can then be modified in some way. Successive applications of this basic process produce a multilevel structure with millions of individual transistors in a square centimeter as described in figure A-1.

Photolithography is the most widely used form of lithography and is likely to continue to be so for the near future. UV light, usually with a wavelength less than 400 nanometers, is used to create patterns resist layers on flat surfaces. Diffraction of light - the interference of light waves with one another-limits the resolution or minimum reproducible feature size. Diffraction is minimized by reducing the wavelength of the exposing radiation. Mercury vapor lamps are routinely used as a source of 436 nanometer (G-line) and 365 nanometer (I-line) light. Shorter wavelength excimer laser sources are a new source of UV light with operation possible at 248 nanometers and in the future 193 nanometers and other wavelengths. By combining shorter wavelengths with improved mask technology (including phase shifted masks ${ }^{2}$ ), better optics, and more sophisticated resist chemistries, it is likely that photolithography will be usable to 0.25 -microns minimum feature sizes and possibly below 0.2 microns.

The longevity of UV lithography is a subject of debate in the semiconductor industry. Many experts argue the limits of diffraction and depth of focus will prevent use of UV photolithography below 0.2 microns and that $\mathrm{x}$-ray lithography, which uses substantially shorter wavelength radiation, will be the successor to UV lithography. $\mathrm{X}$-ray lithography is a candidate for high volume production of integrated circuits with line widths below 0.5 microns. The most studied and developed form of x-ray use is "proximity printing,"

\footnotetext{
${ }^{1}$ H.G. Craighead and M. Skvarla, "Micro and Nanofabrication Technology, Applications \& Impact," contractor report prepared for the Office of Technology Assessment, April 1990; and Tim Studt, "Thin Films Get Thinner as Research Heats Up,' R\&D Magazine, March 1990, pp.70-80.

2Phase shift masks function by carefully controlling light diffraction, using the constructive and destructive interference to helpcreate the circuit pattern. Phase shift masks hold greatest promise for manufacturing memory and other ICs with regular, repeated patterns.
} 
Figure A-1 -Fabrication Sequence for a Metal-Oxide-Semiconductor (MOS) Circuit
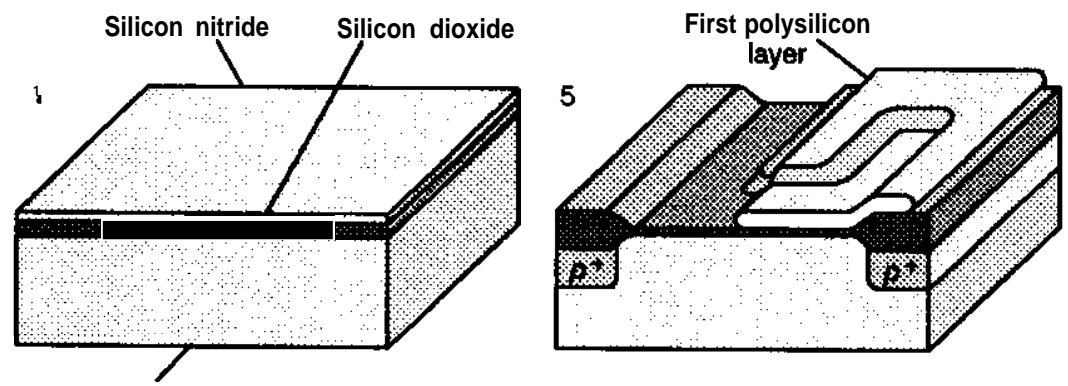

p-Type silicon
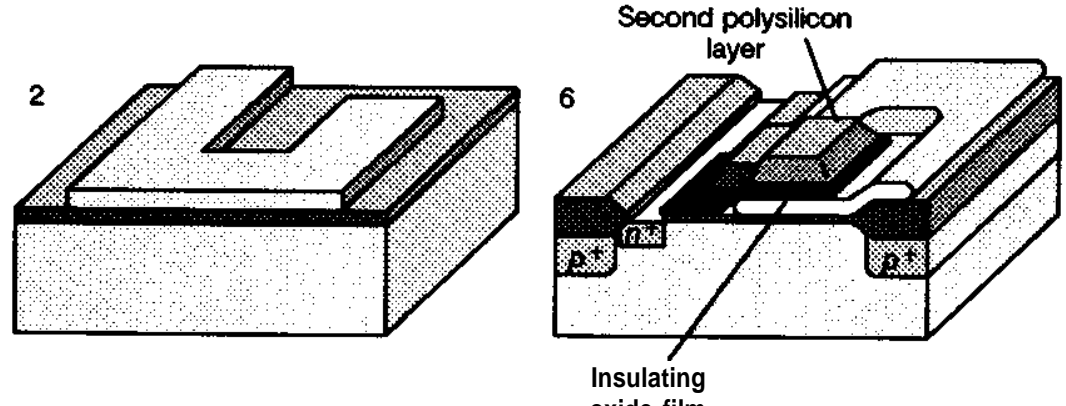

oxide film
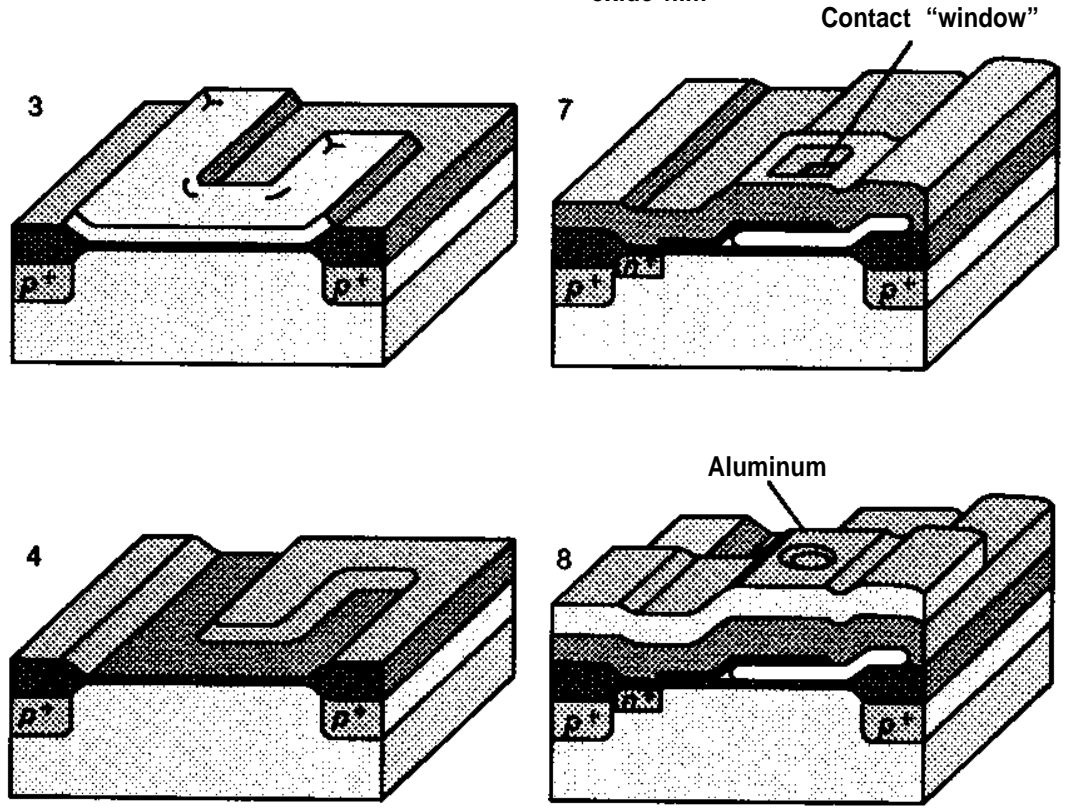

Fabricating this MOS circuit element (a two-level n-channel-negative charge carrier- polysilicon-gate metal-oxide-semiconductor) requires six masking steps. The first few process steps involve the selective oxidation of silicon with the aid of a film of silicon nitride, which acts as the oxidation mask. A thin film of silicon dioxide is grown over the entire wafer, and a layer of silicon nitride is deposited from a chemical (I). The layer is selectively removed in a conventional photolithographic step in accordance with the pattern on the first mask (2). A p-type dopant (e.g., boron) is implanted using the silicon nitride as a mask, followed by an oxidation step, resulting in a thick layer of silicon dioxide in the unmasked-(3). The silicon nitride is then removed in selective etchant that does not attack either the silicon or silicon dioxide (4). Since silicon is consumed in the oxidation process, the thick oxide layer is partly recessed" into the silicon substrate. The first layer of polycrystalline silicon is then deposited and patterned in the second masking step (5). A second insulating film of oxide is grown or deposited, followed by the deposition of the second polysilicon layer, which is in turn patterned in the third masking step (6). A short etch in the hydrofluoric acid at this stage exposes certain regions to an implantation or diffusion of $n$-type dopant. A thin layer of silicon dioxide is deposited next, and contact "windows" are opened with the fourth mask (7). Finally a layer of aluminum is deposited and patterned in the fifth masking operation (8). The wafer will also receive a protective overcoating of silicon dioxide or silicon nitride (not shown); the fact that openings must be provided in this overcoating at the bonding pads accounts for 6 masking steps. Vertical dimensions are exaggerated for clarity.

SOURCE: William G. Oldham, 'The Fabrication of Microelectronic Circuits,” Microelectronics (San Francisco, CA: W.H. Freeman \& Co., 1977), p. 48. Copyright (c) 1977 by Scientific American, Inc.-George V. Kelvin. 
where the mask is placed on the substrate material and its pattern is reproduced by exposure to $\mathrm{x}$-rays, resulting in a substrate pattern the same size as the mask. Practical problems that must be addressed include complexity of mask technologies, difficulty in alignment of multiple layers, economical $\mathrm{x}$-ray sources, and resolution limitations. X-ray lithography operates similarly to UV photolithography, but with much shorter wavelengths-around 5 nanometers. Proximity printing requires a bright source of X-rays. Synchrotron sources are the highest intensity sources of $\mathrm{X}$-rays but they are very expensive. Most experts feel it is unlikely that proximity printing with X-rays will be practical below 0.2 microns, while others hold that the technology will be usable to 0.1 microns.

More recent exploratory work in x-rays involves "reduction projection lithography." X-rays are focused through a mask that is kept away from the substrate. The principal advantages of this approach are cheaper x-ray sources (a synchrotron is not required) and improved resolution. In this country, AT\&T Bell Laboratories and other laboratories are investigating this technology. One of the greatest challenges to making projection x-ray lithography useful for manufacturing is the optics used in the process; they are complex, involving the creation of multiple-layer films with precisely controlled thickness.

Another way to get beyond the diffraction limit of radiation-based lithography is to use electrons or atoms to expose the resist. Diffraction does not limit the resolution of electron-beam lithography because the quantum mechanical wavelengths of high energy electrons are exceedingly small. Electrons scatter quickly in solids, limiting practical resolution to dimensions greater than 10 nanometers-significantly greater than current demands of any practical technology. Electron-beam lithography has demonstrated resolution as small as 2 nanometers (0.02 microns) in a few materials. Electron beam technology, however, is limited in usefulness because an electron beam must be scanned across the entire wafer. Electron-beam lithography tools are in use in universities, in laboratories, and in industry for mask-making and small manufacturing production runs.

There are approaches still in research that may yield a more versatile lithography tool. A possible alternative is to use photolithography for large feature definition and reserve the scanned electron beam for the critical dimensions. A varietyof approaches for parallel exposure, rather than serial scanned exposure, of electron-beams have been studied for years. There is currently active research in electron beams reduction projection using masks at AT\&T Bell Laboratories, and in multiple source systems and proximity printing at IBM. These approaches would exploit the resolution and alignment possibilities of electron beams with the speed of parallel exposure techniques.

Ion beam lithography is in many ways similar to electron beam lithography with beams of charged atoms (ions) taking the place of electrons. Recent advances in ion sources have increased the utility of scanned focused ion beams. Compared to photons (x-rays and light) or electrons, ions chemically react with the substrate, allowing a greater variety of modifications. Some scattering effects are also reduced compared to electrons and, as with electrons, diffraction limits of ion beams are negligible. The fundamental resolution limits for charged particle lithographies are below 1(M) nanometers. Ion beam lithography suffers the same draw back as electron beam systems; it relies on a serially scanned beam. That limits its applications to products that do not require high throughput-e.g., masks and small batches of electronics. Research efforts are underway to develop parallel projection and multiple source ion beam lithographic equipment that might overcome the limitations of serially scanned ion beam lithography.

Scanned probe modification of surfaces, using recently developed scanning tunneling microscopy (STM) methods, is being done by researchers in many research laboratories. Since STMs can manipulate individual atoms, such approaches have theoretical resolution limits of single atoms. This approach is still in early phases of research and is focusing on basic physics measurements 
and better understanding of the behavior of surfaces. The lack of stability of STM tips and slow speed, however, make it far from a practical technology for manufacturing in the foreseeable future.

\section{PATTERN TRANSFER}

The various lithography systems produce relief patterns in resists, and a subsequent pattern transfer step is necessary to fabricate the structure. There are a number of options available and each involves some engineering tradeoffs. Once the resist pattern is in place, and selected areas of the underlayer are exposed, there are three possible next steps: 1) add to, 2) remove, or 3) modify the exposed areas.

Adding to the exposed area involves either deposition or growth of a material on the exposed areas. Growth is different from deposition because it involves consumption of the surface (usually combining with some introduced chemical) to create the new substance. The most common example is a silicon surface being consumed in the process of forming silicon dioxide. It involves heating the silicon wafer in the presence of oxygen, causing the growth of silicon dioxide from the exposed silicon. A number of materials are grown in this way, since the consumption of the surface results in excellent adhesion, better electrical and mechanical properties, and improvements in other materials properties. This specific reaction has been exhaustively investigated and perfected and now can be used to grow oxide films whose thickness, and even lateral dimensions, can be measured in atomic layers (a few angstroms).

Deposition creates new layers of material on the exposed area. These techniques usually involve evaporating or sputtering a piece of material-the target-so its atoms or molecules fly off and land on the sample. Deposition techniques include:

- spin-on,

- thermal evaporators,
- sputtering,

. laser ablation deposition,

- chemical vapor deposition; and

- molecular beam epitaxy such as organometallic chemical vapor deposition.

Spin-on deposition is the simplest deposition technique. It involves spinning the sample while a liquid is poured onto it; the spinning action distributes the liquid evenly. Subsequent heating bakes the liquid into a solid thin film.

Thermal evaporators use a heated filament or an electron beam to vaporize material. The material passes through a vacuum to impinge on the sample, building up a film. Evaporators emit material from a point source, resulting in "shadowing" and sometimes causing problems with very small structures.

Sputter deposition systems erode atoms from a broad target that then travel to the sample. This results in better line width control and uniformity, especially with high aspect ratio (height/width) structures. Refinements of these processes, e.g., in situ cleaning with an ion gun, or using electron cyclotron resonance to confine and densify a plasma, result in better adhesion, higher quality films, and more versatility. Using lasers or particle beams in conjunction with deposition presents the possibility of defining patterns on the sample in a single step.

Laser ablation deposition uses intense laser radiation to erode a target and deposit the material onto a substrate. This technique is particularly useful in dealing with compounds of different elements —e.g., yttrium-barium-copper oxide superconductor films.

Chemical vapor deposition (CVD) deposits thin films by passing reactive gases over the sample. The substrate is heated to accelerate deposition. CVD is used extensively in the semiconductor industry and has played an important role in past transistor miniaturization by making it possible to deposit very thin films of silicon. Most CVD is performed in vacuum, but new techniques allow operation without vacuums. Radio frequency $(\mathrm{RF})$ or photon radiation can be used 
to enhance the process and is known as plasma enhanced CVD (PECVD).

Some deposition techniques are so precise that material can be built up literally atom by atom with the crystal structure of the new material exactly matching that of the underlying layer. In molecular beam epitaxy (MBE), the sample is placed in an ultra high vacuum in the path of streams of atoms from heated cells that contain targets of various types. These atomic streams impinge on the surface, creating layers whose structure is controlled by the crystal structure of the surface, the thermodynamics of the constituents and the sample temperature. Organo-metallic chemical vapor deposition (OMVPE, or sometimes MOCVD) relies on the flow of gases (hydrides like arsine and phospine or organometallics like tri methyl gallium and tri methyl aluminum) past samples placed in the stream. Again, the sample surface and thermodynamics of the processes determine the compounds deposited. Both MBE and OMVPE provide thickness control within one atomic layer (a few angstroms) and are especially useful in creating compound semiconductors and exploiting quantum effects and band-gap engineering. This structural control enables researchers to exploit optical transitions in some materials, producing lasers, detectors, and other optical elements.

Materials modification processes are used mainly to vary the electrical conductivity in the appropriate areas. In the past, dopants (atoms that can either contribute or subtract electrons from silicon atoms) were diffused into the substrate thermally; now they are implanted as high energy ions (see figure A-2). Implantation offers the advantage of being able to place any ion at any depth in the sample, independent of the thermodynamics of diffusion and problems with solid volubility and precipitation. Ion implantation, originally developed for high energy physics, is now an indispensable part of semiconductor manufacturing. Ion beams produce crystal damage in addition to the chemical or electronic effect of the dopants. Since crystal damage reduces electrical conductivity, this effect can be exploited to electrically isolate devices from one another. Ion beams can implant enough material to actually form new materials--e.g., oxides and nitrides-some of which show improved wear and strength characteristics.

Subtractive processes - the removal of material-are also vital to the field. Some of the steps are still done the same way they were centuries

Figure A-2-ion Implantation
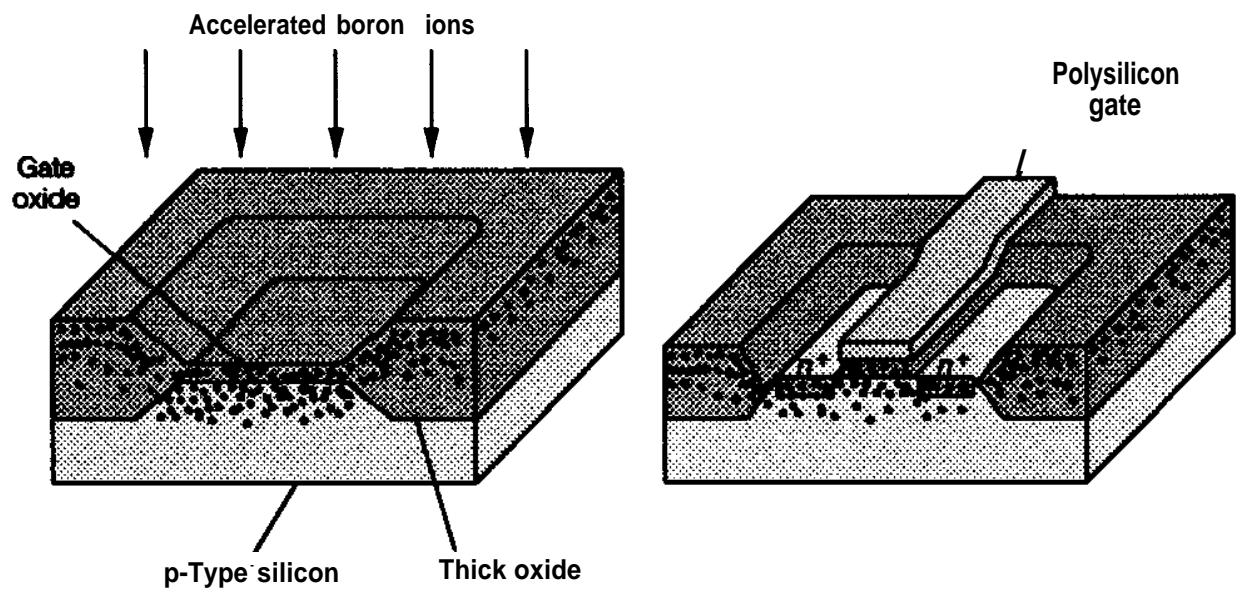

Ion implantation is employed to place a precisely controlled amount of dopant (in this ease boron ions) below the gate oxide of a MOS transistor. By choosing a suitable acceleration voltage the ions can be made to just penetrate the gate oxide but not the thicker oxide (left). After the boron ions are implanted polycrystalline silicon is deposited and patterned to form the gate regions of the transistor. A thin layer of the oxide is then removed and the source and drain regions of the transistor are formed by the diffusionof an $n$-type impurity (right).

SOURCE: William G. Oldham, "The Fabrication of Microelectronic Circuits," Microelectronics (San Francisco, CA: W.H. Freeman \& Co., 1977), p. 50. Copyright (c) 1977 by Scientific American, Inc. -George V. Kelvin. 
ago. The object, with its lithographically defined areas exposed and the remainder protected by the "resist," is dipped into a chemical bath and a reaction or dissolution is allowed to take place. Unfortunately, liquid chemicals usually have no sense of direction and will dissolve at an equal rate on all sides. As device geometries shrink, it becomes important to control directionality. Most modern etching is done in vacuum without contact with liquids.

Ion milling uses the sputtering process to bombard the substrate with accelerated ions. The sputtering process relies on a physical mechanism whereby energy is transferred to a surface by impinging ions. This energy is enough that some atoms break free of the surface. This mechanism erodes all materials, with small differences in rate between any two. Selectivity is poor, and any masking material (resist) will disappear as fast as the underlayer, limiting the (relief) depth.

Reactive ion etching (RIE) can be highly selective because it is a chemical reaction between ions (radicals) formed in a plasma and atoms on the sample surface, and can be highly selective. RIE has excellent lateral control because the electric fields produced by the plasma cause ions to diffuse directionally. Straight parallel trenches a micron or more deep can be etched without affecting a rather fragile resist mask; minimum feature sizes of a few nanometers are possible. RIE is highly selective in its etching: oxygen etches hydrocarbons (resists), fluorine compounds are useful in working with silicon systems, and many materials are susceptible to chlorine etch, especially the compound semiconductors like gallium arsenide. A great deal of work is directed at discovering the etching characteristics of other compounds.

The combination of physical and chemical etching is achieved in a process called chemically assisted ion beam etching (CAIBE). The combined effects of ion sputtering and chemical reaction with reactive ions results in a faster etch rate than that due to either mechanism alone. CAIBE is of particular interest to research groups inves- tigating new materials, since it can be exploited to uncover ways to etch new and exotic materials.

Unlike ancient lithographs, modern semiconductors must undergo many processing steps. The mask must remain intact until the substrate etch is complete, without changing feature size or shape or surface characteristics (morphology). Multiple operations are often required because of the processing limitations of materials. For example, to grow a thin layer of silicon dioxide at $1,000^{\circ} \mathrm{C}$, a resist is used to pattern a silicon nitride layer which is then capable of surviving the high temperature during the oxide growth. Multiple layers are often processed in succession to better allow a straightedge to be carried through a thick stack of material.

\section{CHARACTERIZATION}

Characterization has become an indispensable tool in the art and science of fabrication. The designer needs information about device performance, the experimenter needs to know composition and concentration, and the process engineer must have immediate confirmation of the electrical, mechanical, optical, or chemical properties of a thin film. In fact characterization, or failure analysis, is in some cases as difficult and extensive as the original experiment. The most common questions addressed by characterization concern the shape and size of the structure. Optical and electron microscopes provide this information over size ranges from hundreds of microns to fractions of a nanometer (near-atomic dimensions).

Instruments also exist to measure a range of materials properties, including the thickness, refractive index, and electrical resistivity. While these techniques involve the measurement of thin film properties over large distances, microcharacterization can determine composition or structure on a size scale comparable to the mean free path of an electron and some techniques have an ultimate resolution on the order of a few atomic diameters. 
There are a wide range of techniques usually identified by acronyms, and some of the more useful are listed here:

\section{SCANNED BEAM TECHNIQUES}

\begin{tabular}{|c|c|}
\hline SEM - & $\begin{array}{l}\text { Scanning electron microscope: } \\
\text { creates image of a surface by } \\
\text { measuring the reflection from a } \\
\text { beam of electrons. }\end{array}$ \\
\hline TEM - & $\begin{array}{l}\text { Transmission electron micro- } \\
\text { scope: determines crystal struc- } \\
\text { ture by measuring transmission } \\
\text { of electrons through a thin sam- } \\
\text { ple; has high resolution. }\end{array}$ \\
\hline
\end{tabular}

AES - $\quad$ Auger electron microscopy: identifies constituents in the surface layer by measuring energy of electrons emitted due to "Auger transitions."
RBS - $\quad$ Rutherford backscattering spec- troscopy: identifies constituents by measuring the spectrum of scattered ions off a sample.

SIMS - $\quad$ Secondary ion mass spectroscopy: analyzes composition of material removed by ion bombardment.
XRF - $\quad$ X-ray fluorescence: identifies composition from photon emis- sion due to x-rays.
EDS (EDX) - Energy dispersive X-ray spectros- copy: identifies composition from dispersion of $\mathrm{x}$-rays.
IMMA -
Ion microprobe mass analysis: high resolution secondary ion mass spectroscopy (SIMS).
EELS - $\quad$ Electron energy loss spectrosco- py: identifies composition by measuring energy loss of elec- trons during electron interaction.
ED - $\quad$ Electron diffraction: measures crystal structure by diffraction of electrons.
LEED - Low energy electron diffraction: measures surface structure by diffraction of electrons.
RHEED - Reflection high energy electron diffraction: measures surface structure; particularly useful for epitaxial film growth.

\section{SCANNED PROBE TECHNIQUES}

STM -

AFM -

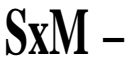

Scanning tunneling microscopy: creates image of surface using electron tunneling between the surface and a sharp tip near the surface; very high resolution.

Atomic force microscopy: creates image of surface using repulsion of electron charge distributions between surface and a tip; nearatomic resolution is possible.

Other developing scanned probe microcopies: create images of surface characteristics for magnetic force, electrochemical measurement, etc., using the principle of the STM. 
Actuator: Causes mechanical force or motion in response to an electrical signal.

Analog: Refers to electrical signals that can vary continuously over a range, as compared to digital signals, which are restricted to a pair of nominally discrete values.

Angstrom: One tenth of a nanometer, $10^{-10}$ meters.

Architecture: The overall logic structure of a computer or computer-based system.

Capacitor: Passive circuit element that stores electrical charge, creating a voltage differential. Capacitors can be fabricated within integrated circuits, as well as in the form of discrete components.

Charge-Coupled Devices (CCD): A type of solidstate electronic device used as a sensor in some types of cameras.

Circuit Board: A card or board of insulating material on which components such as semiconductor devices, capacitors, and switches are installed.

Clock An electronic circuit, often an integrated circuit, that produces high-frequency timing signals. A common application is synchronization of the operations performed by a computer or microprocessor-based system. Typical clock rates in microprocessor circuits are in the megahertz range, 1 megahertz equaling $10^{6}$ cycles per second.

Compact Disk (CD): An optical storage medium used for music and for computer data, among others.

Deposition: An operation by which a film is placed on a surface.

Die Bonder: Assembly equipment that bonds the back side of an integrated circuit die to various materials. A die is a small piece of silicon wafer that contains the complete circuit being manufactured.
Doping: Adding to a semiconducting material small amounts of other elements (dopants) to change its electronic properties.

Dynamic Random Access Memory (DRAM): The most common type of computer memory. DRAM architecture usually uses one transistor and a capacitor to represent a bit, which is a memory cell in a computer.

Electron Cyclotron Resonance (ECR): A technology that uses a high-frequency microwave energy source to create a plasma in a confined region using a magnetic field for the purpose of etching and deposition.

Etching: A process in which chemicals are used to remove previously defined portions of the silicon oxide layer covering the wafer to expose the silicon underneath.

Flat Panel Displays: A thin display screen that uses any of a number of technologies, such as liquid crystal display. Flat panel displays are used in laptop computers in order to keep the overall size and weight of the machine to a minimum.

Gallium Arsenide (GaAs): A compound semiconductor with properties necessary for very high-frequency microwave (analog) devices and optoelectronics.

Gate: A simple electronic circuit that can implement a specified logical operation. In essence, gates act like switches. Computer processing units depend on large assemblies of gates, as do integrated circuit memory chips.

Integrated Circuit (IC): Electronic circuits, including transistors, resistors, capacitors, and their interconnections, fabricated on a single small piece of semiconductor material (chip). Categories of ICs such as LSI and VLSI refer to the level of integration, which denotes the number of transistors on a chip. ICs may be digital (logic chips, memory chips, or microprocessors) or analog. 
Ion Implantation: A process in which silicon is bombarded with high-voltage ions in order to implant them in specific locations and provide the appropriate electronic characteristics.

Liquid Crystal Display (LCD): A liquid crystal display is a technique that uses a transistor for each monochrome or each red, green and blue dot. It provides sharp contrast and speeds screen refresh. LCD technology is commonly used in digital watches and laptop computers.

Lithography A process in which the desired circuit pattern is projected onto a photoresist coating covering a silicon wafer. When developed, portions of the resist can be selectively removed with a solvent, exposing parts of the wafer for etching and diffusion.

Logic Chips: ICs that manipulate digital data.

LSI: Large-scale integration. Typically involving between 2,000 and 64,000 transistors.

Mainframe Computer: A system, normally intended for general-purpose data processing, characterized by high performance and versatility. Mainframes have grown steadily in capability as smaller and less expensive machines have progressed.

Mask: Stencil-like grid used in creating lithographic patterns on semiconductor chips.

Mass Storage: Refers to peripheral equipment for computer memory suitable for large amounts of data or for archival storage. Typical mass storage devices are disk and tape drives.

Memory Chips: Devices for storing information in the form of electronic signals.

Microcomputer: Refers to integrated circuits that contain a microprocessing unit plus memory, as well as to computers designed around microprocessors or single-chip microcomputers.

Micrometer: One-millionth of a meter.

Micron: One-millionth of a meter.
Microprocessor: A computer central processing unit on a single chip.

Nanometer: One-billionth of a meter.

Optical Disks: Recording media including CDs that store information in patterns of microscopic pits on the surface of the disk, which can then be detected by a solid state laser and detector system and reproduced as sound, images, or data.

Optoelectronic Devices: Devices that convert light signals to electronic signals and vice versa.

RAM (Random Access Memory): Most commonly, an integrated circuit that stores data in such form that it can be read, erased, and rewritten under the control of a computer processor. Any memory location in a RAM can be addressed directly (random access) as opposed to sequentially or serially.

Resist, Photoresist: Chemicals used in lithographic processing of integrated circuits which, much like photographic emulsions, can be exposed by light, X-rays, or other radiation to form patterns.

Semiconductors: Materials, e.g., silicon, that have four electrons in their outer electron shells and have electrical conductivities that are lower than good conductors such as metals but higher than insulators such as glass. Other semiconductors include germanium, gallium arsenide (GaAs), iridium phosphide (InP), mercury telluride(HgTe), cadmium telluride (CdTe), and alloys of these compound semiconductors.

Sensor: Converts a pressure, temperature, or other physical parameter into an electrical signal, often for use in a control system. A digital speedometer for an automobile transforms the output of a sensor into a miles-per-hour reading, as does an airplane's air speed indicator. In the case of the automobile speedometer, rotary motion is converted into an electrical signal, while an air speed indicator depends on the pressure created by the motion of the airplane. 
Silicon: One of the most common elements found in nature and the basic material used to make the majority of semiconductor wafers.

Stepper: A sophisticated piece of equipment used to transfer an integrated circuit pattern from a mask onto a wafer.

Substrate: A piece of material, typically a semiconductor, on which layers of materials are deposited and etched to fabricate a device or a circuit.

Transistor: An electronic device that can be used to switch or amplify electronic signals.

VLSI: Very large-scale integration. Typically involving more than 64,000 transistors.
Wafer: A thin disk, cut from silicon or other semiconductor material. The wafer is the base material on which integrated circuits are fabricated. It is typically 4 to 8 inches in diameter.

Yield: In the production of microelectronic devices, the fraction that survive all tests and inspection, function correctly, and can be sold or incorporated into the manufacturer's own end products. Production costs depend heavily on yields, which themselves depend on circuit design, fabrication equipment, and control of the manufacturing process. 
Accelerometer, 1,30-31

Analog Devices, Inc., 9,31

Angstrom technology, 7

Arthur D. Little, Inc., 28

Assemblers, 20,21

AT\&T Bell Laboratories, 7,9, 11,31,33,37

Automobiles, 3,4,9

Biosensors, 3,8,28-30

Blood analysis, 29

Bosch, 10

Bulk micromachining, 31

\section{California, 9}

Canon, 33

Cellular automata, 16

cellular phone, 5

Characterization, 3,35,40-41

Charge coupled device, 27-28

Chemical field effect transistor, 28

Chemical sensors, 3,8,28-30

Chemical vapor deposition, 38

Chemically assisted ion beam etching, 40

chip size, 14,22,24

Clark, Leland C., 28

Clock cycle, 4, 15,24

Communications, 5, 16

Consumer electronics, 4,5, 16,26,27,28,33

Defense Advanced Research Project Agency, 7,9, 11

Department of Defense, 7,8,9,28

Department of Energy, 9

Digital Equipment Corp., 7

Display technology, 5,23

Doping, 39

Drexler, Eric, 21

Dynamic random access memory, 1,4,5,13,15

Electron-beam lithography, 11,13, 17,37

Etching, 39-40

Eutaxic control, 20

Feynman machines, 20

Feynman, Richard, 21

Fiber-optics, 29

Flat panel display, 5,23

Fluid flow, 31,33

Food processing, 28

Fraunhofer Institute, 9, 10

Fujitsu, 7

Gallium arsenide, 16, 17

German Federal Ministry for Research and Technology, 9

German Karlsruhe Nuclear Research Center, 9, 10,31

Glucose sensor, 28,29-30

Hewlett-Packard, 33

High electron mobility transistor, 16
High-performance computers, 15,26

Hitachi, 33

Hybrid wafer-scale integration, 24

Interconnection, 8, 13, 15, 17,21,23-27

International Business Machines, 7, 10, 11,23,24,37

Ion-beam lithography, 11, 17,37

Ion implantation, 39

Ion milling, 40

Japanese Basic Technologies for the Future Industries Program,

Japanese Exploratory Research for Advanced Technology, 7

Japanese Large Scale Program, 7

Japanese Ministry of Agriculture, Forestry and Fisheries, 8

Japanese Ministry of International Trade and Industry, 7,33

Japanese Science and Technology Agency, 10

Japanese Technology and Evaluation Center, 8

Jet Propulsion Laboratory, 33

Kodak, 28

Laptop computer, 5,19

Lasers, 16, 18,23,38

Lawrence Livermore National Laboratory, 9,33

LIGA, 31

Lithography, 10-11, 13, 17-19,23,31,32,35-38

Louisiana, 9, 11

Louisiana Technical University, 9

Magnetic storage, 23

Mainframe computer, 19

Manufacturing, 3, 13,30,32

Manufacturing costs, 1,3, 13

Mass storage, 17,23

Massachusetts Institute of Technology, 31

Materials science, 3, 16, 19,31,4041

Matsushita Central Research, 10

Mechanical properties, 31

Medical applications, 16,28-30,33

Messerschmidt, 10

Micro-Electromechanical Sensors and Actuators Institute, 10

Microelectronics and Computing Technology Consortium, 8,24

Micromachining, 30

Micromechanics, 1,3,8,9,20,30-34

Micromotors, 32-33

Microprocessor, 4

Microrobots, 10,33

Military applications, 16

Mitsubishi, 17

Molecular beam epitaxy, 16,38,39

Molecular electronics, 3,7, 16, 17

Molecular machines, 20-21

Motorola, 11

Multi-chip modules and packages, 4,8,24,25

Nanobots, 20

Nanotechnology, 20 
National Aeronautics and Space Administration, 9,33

National Institutes of Health, 9

National Research Council, 8

National Science Foundation, 7,9

Netherlands, 10

New York, 11

Nippon Electric Co., 5, 10,24

Nippon Telephone \& Telegraph, 7, 10

Noise, 15

Optical storage, 17,23

Optics, 16,33,39

Optoelectronics, 7,39

Organo-metallic chemical vapor deposition, 39

Packaging, 4,5,23-27,32

Phase-shift lithography, 10, 19,35

Pressure sensor, 30

Quantum electronics, 3,7,15,16

Reactive ion milling, 40

Refrigeration, 15, 19,26

Ricoh, 10

Sacrificial layer, 31-32

Sandia National Laboratory, 9

scaling laws, 15

Scanning tunneling microscope, 19,20,23,37,41

Semiconductor manufacture g equipment, 1,3,4,9, 10-11, $17-19,30,31-32,35-41$
Siemens, 10

Space applications, 16

Spin on, 38

Sputtering, 38

Surface micro-machining, 31-32

Surface mount technologies, 4,6,8

Surface science, $3,19,31,38,40-41$

Switzerland, 10

Tektronix, 28

Texas Instruments, 7,28

Thermal evaporation, 38

Toyota, 10

Traditional machining, 33

U.S. Congress, 9, 11

Ultraviolet lithography, 10, 17,31,35,37

University of California at Berkeley, 31,33

University of Delft, 10

University of Pennsylvania, 29

University of Tokyo, 10

University of Twente, 10

University of Utah, 33

von Neumann architecture, 16, 27

X-ray lithography, 10, 11, 13, 17,31,32,35,37 